\title{
Decision Making Under Uncertainty: Applying the Least-Squares Monte Carlo Method in Surfactant-Flooding Implementation
}

\author{
A. Alkhatib, M. Babaei, and P.R. King, Imperial College London
}

\begin{abstract}
Summary
This study introduces a decision-making evaluation method for flexibility in surfactant flooding. The method aims to capture the effects of uncertainty in the time series for both technical and economic parameters and produce a near-optimal policy with respect to these uncertainties as they vary with time. The evaluation method used was the least-squares Monte Carlo (LSM) method, which is best-suited for evaluating flexibility in project implementation. The decision analyzed was that of finding the best time to start surfactant flooding during the lifetime of a field under uncertainty. The study was conducted on two reservoir models: a 3D homogeneous model and a 2D heterogeneous model. The technical uncertainties considered were the residual oil saturation (ROS) to the surfactant flood, surfactant adsorption, and reservoir heterogeneity, and the main economic uncertain parameters considered were oil price, surfactant cost, and water-injection and -production costs. The results show that the LSM method provides a decision-making tool that was able to capture the value of flexibility in surfactant-flooding implementation and provides some insight into the effect of uncertainty on decision making, which can help mitigate adverse circumstances should they arise or capture the upside potential if circumstances prove beneficial. The results found that the optimal policy obtained was reliable and that heterogeneity and different well-placement patterns affect the value of flexibility and optimal policy for different reservoir models. Furthermore, possible extensions to enhance the LSM method were discussed.
\end{abstract}

\section{Introduction}

Enhanced-oil-recovery (EOR) processes have received increasing attention lately because of high oil prices and maturing giant fields around the world. Chemical EOR represents a small part of the picture. Wide-scale implementation of surfactant flooding faces many challenges that arise mainly because of uncertainty in field implementation (Costa et al. 2008). This uncertainty is a result of various factors such as heterogeneity and surfactantrelated rock properties. Our understanding of uncertainty changes with time, depending on the evolving state of knowledge because of data acquisition, coreflooding, production history, and new surveillance technology. Therefore, it is sufficient to assume that uncertainty is evolutionary. To implement a successful surfactant field project, a better understanding of how to manage the evolutionary state of uncertainty is required to enhance the decisionmaking process. This study introduces a decision-making evaluation method for flexibility in surfactant flooding that can be applicable to other EOR processes. The value of flexibility is defined as the value of splitting decisions into multiple decisions over time, with the opportunity to learn between decisions and having the option to respond to that learning (Bratvold and Begg 2010).

Copyright $(C 2013$ Society of Petroleum Engineers

This paper (SPE 154467) was accepted for presentation at the EAGE Annual Conference \& Exhibition incorporating SPE EUROPEC 2012, Copenhagen, Denmark, 4-7 June 2012, and revised for publication. Original manuscript received for review 3 February 2012 . Revised manuscript received for review 18 January 2013. Paper peer approved 23 January 2013.
The LSM method was used to evaluate and determine an optimal policy in implementing an EOR process, specifically a surfactant flood. The method aims to capture the effects of uncertainty in both the technical and economic time series and produce an optimal policy with respect to these uncertainties as they vary with time. The method was applied to two synthetic reservoir models: a 3D homogeneous model and a 2D heterogeneous model. The paper is structured as follows. The first section provides a brief overview of previous work that focused on the decision-making evaluation processes used in EOR and, more specifically, surfactant-flooding implementation. The second section will define the problem (decision) that we want to analyze. The LSM method is then introduced, with its components discussed in further detail. The fourth section will introduce the results and discussion of the study; finally, the conclusions are presented.

\section{Decision-Making Evaluation Processes for Surfactant Flooding}

Decision-making methods have been used in the industry since 1962 (Grayson 1962). These methods have been advantageous because of the highly uncertain nature of many activities in the industry. Because we are always faced with limited resources, this necessitates that we use these resources in an optimal (or nearoptimal) manner to produce the best decisions given the information available at the time of the decision. Barua et al. (1986) introduced one of the earliest decision-making methods in surfactant flooding, a way to optimize the technical decisions first (such as slug size and concentration) and then incorporate an economic model to produce the objective function, the net present value (NPV). The engineering decisions it solved for were the size and concentration of the surfactant slug, the size of the polymermobility buffer, and the number of infill wells to be drilled. The aim of the optimization is to determine the decision-variable values that maximize the objective function, such as the NPV or some other parameter. Economic risks were treated deterministically by considering a few cases of oil prices that were held constant throughout production.

Sanz and Miller (1994) applied decision analysis to a chemical-flood design on the basis of data from the pilot test at the Big Muddy field, Wyoming. It used the US Department of Energy (DOE) MCPALK3 model (DOE 1986) as the surfactant-flooding simulator. Sensitivity analysis was used to determine which state and control variables had the greatest effect. Surfactant concentration, injection rate, surfactant-slug size, polymer-slug size, and pattern area were the most significant decision variables; ROS was found to be the most influential state variable. Afterward, Monte Carlo simulation was performed to study the effects of uncertainty on the objective function. Two objective functions were used: For deterministic modeling, the profit-to-investment ratio was chosen, and for stochastic modeling, the objective function was the NPV.

Manrique et al. (2009) proposed a decision-making workflow that emphasizes the use of screening techniques in making business decisions in an EOR context. They presented two types of field cases: cases that had limited data and limited decision time, 
and cases that faced only limited-time scenarios. The screening approaches relied on fuzzy logic, artificial intelligence, and datamining techniques. Performance prediction relied mostly on simplified numerical or analytical models because of the time constraints presented in the decision cases. Economic risk was treated from a deterministic outlook. One of the cases considered presented uncertainty in heterogeneity, which was analyzed with the Dykstra-Parsons coefficient. It considered various field lithologies and EOR methods. Their results supported flexible decision-making workflow that can be adaptable to different volume and quality of information. They also argued that a model should be only as complex as necessary to support rational decision making. Complexity of the decision analysis has not greatly contributed to improving decision making in the industry (Bickel and Bratvold 2007). One of the disadvantages of this approach was that screening relied greatly on a database of previous EOR projects, which might not be available to everyone.

Costa et al. (2008) introduced a method to quantify the impact of chemical uncertainties related to alkaline/surfactant/polymer flooding, to improve the sweep and displacement efficiency in a Brazilian onshore field. This study focused on use of representative models in decision making for an alkaline/surfactant/polymer flood. This approach was rooted in finding the parameters with the highest impact on performance through sensitivity analysis and then obtaining a risk curve that is similar to a probability distribution. Then, three representative models were chosen to represent the P10, P50, and P90 cases. Economic uncertainty was then integrated with the representative models to improve decision making. Performance prediction was numerically simulated with STARS/CMG to evaluate field performance. The study focused on selecting and using representative models to provide probabilistic scenarios that can represent the chemical and dynamic uncertainties to be able to integrate them with economic uncertainties to improve decision making. However, the study did not take into account uncertainty in heterogeneity.

After reviewing decision-making models, it is noteworthy to also review traditional valuation methods of surfactant flooding or other EOR processes that could be easily used for surfactant flooding. This is to provide a more complete picture of how these models are designed and how the LSM method could be more robust with respect to managing uncertainty and its influence on project value. Traditional valuation of EOR projects was modeled after discounted-cash-flow methods that did not take into account uncertainty in the time series of the economic and technical state variables and its influence on the success of a an EOR project (Trantham 1983; Gittler and Krumrine 1985; Tomich et al. 1987; Anderson et al. 2006; Wyatt et al. 2008). Thus, it produced a project value on the basis of fixed realizations. These models do not capture the effects of uncertainty and therefore cannot capitalize on upside opportunities or mitigate downside risk. When considering uncertainty in technical or economic variables, these methods then assessed the value of an EOR project on different combinations of the values of these variables (usually a lower bound, median, and upper bound) or they assumed that these variables obey a specific distribution (e.g., uniform or normal) from which they are sampled and then they are assumed to be fixed during the life of the project. Therefore, these methods produce deterministic values on the basis of fixed realizations of state variables and strategies.

To develop optimal policies for surfactant-flooding EOR, we need to be able to make decisions taking into consideration the uncertainty in technical and economic parameters. Furthermore, we need to be able to incorporate the uncertainty in the time series of these parameters caused by our evolving state of information over time. None of the previously reviewed models addresses all these issues in their entirety. The LSM method is proposed as a decision-making algorithm that can provide optimal policies and can capture the effects of uncertainty and flexibility. This method requires a well-defined policy (a control variable, such as how much surfactant to inject or when to inject, or a combination of different controls), which is intended to be optimized.

\section{Problem Definition}

The objective of this study was to maximize the value of a surfactant flood by creating value from uncertainty and flexibility through capturing the effects of technical and economic risks. Our information set will vary with time because we assume for the examples considered in this study that there is an uncertaintyreducing process that is ongoing from an external source of information, such as corefloods or alternative pilots. However, this is not always the case in reality. The problem we will investigate in this study is that of surfactant-flooding initiation within a certain time frame. We assumed a field life of 10 years, and we want to determine the best time to start surfactant flooding for this field. The option here is whether to begin a surfactant flood at the start of the fourth, fifth, sixth, or seventh year of the field life. Therefore, we assume a decision space, $Y=(4,5,6,7)$, and a decision node, $y_{n} \in Y$. These decision nodes were chosen because the water breakthrough of the two models investigated in this study occurred either a little before or a little after the fifth year. We decided to investigate whether it was best to begin a surfactant flood before, during, or after water breakthrough. This defines our options scenario. A no-option scenario is assumed to begin surfactant injection at the start of the field life. This was chosen through initial deterministic case simulations (these runs used a surfactant-adsorption value of $10^{-4} \mathrm{lbm} / \mathrm{lbm}$ rock and $0 \%$ ROS to chemical flooding) for the two reservoir models used and it will form the basis of our analysis when evaluating optionality. Clearly, there will be a bias in the results depending on our choice of the no-option scenario. The reservoir state variables were assumed to be the ROS to surfactant flooding $\left(S_{\text {orc }}\right)$ and the surfactant adsorption $\left(D_{s}\right)$. The economic state variables were assumed to be the oil price $\left(P r_{o}\right)$, surfactant cost $\left(P r_{s}\right)$, water-injection cost $\left(P r_{w i}\right)$, and water-production cost $\left(P r_{w p}\right)$.

There are a number of technical considerations that can influence the surfactant-flood design, such as conformance strategies, mobility control, chemical treatment, mixing requirements, and water source (Trantham 1983; Raterman et al. 1988; Reppert et al. 1990; Maerker and Gale 1992). These issues are not considered as part of the decision strategy in this study because our main aim is to demonstrate the LSM method in decision making in the context of surfactant flooding. These issues are essential for any detailed field or reservoir study and will be included in the decision-making method for more-complex scenarios. Furthermore, we have assumed a fixed set of control variables (e.g., slug size, surfactant concentration) to simplify the decision process and demonstrate the method. In reality, practical considerations of the control strategies that take into account field or reservoir peculiarities must be included in the decision process. This would include, for instance, varying the surfactant-slug properties (volume or concentration) as the reservoir conditions change with time.

\section{LSM Method}

The LSM method provides a framework for evaluating options as a function of the relevant uncertainties. It produces a dynamic outlook rather than a static outlook, in which uncertainty introduces opportunities as well as risk. The motivation behind the use of this approach is that by designing flexibilities into a process, resolution of uncertainties over time provides opportunities to change course and create value. Valuation methods should capture the relationship between project uncertainties and market movements (Jafarizadeh and Bratvold 2009). LSM is useful when decisions are dependent on multiple uncertain factors and when compounded options exist (Willigers and Bratvold 2009). These decisions (or options) can be valued as conditional on the full or partial resolution of the relevant uncertainties with time. The main challenge is, given these uncertainties, how to evaluate the value of a decision or option.

This approach is derived from a forward-looking Monte Carlo simulation and a backward recursive dynamic programming technique that evaluates the option (Gamba 2002). A good understanding of the decisions or problems is needed to implement 
TABLE 1-RANGE ADJUSTMENT VALUES FOR $S_{\text {orc }}$ AND $D_{s}$ STOCHASTIC MODELS

\begin{tabular}{|lcccc|}
\hline$t$ & 1 & 2 & 3 & 4 \\
\hline$q_{t}$ & 0.4 & 0.3 & 0.2 & 0.1 \\
$q_{d t}$ & 0.2 & 0.1 & 0.05 & 0.01 \\
\hline
\end{tabular}

LSM successfully, and good choice of basis functions is also necessary (Jafarizadeh and Bratvold 2009).

The LSM method is performed by

- Generating a large number of possible realizations of the state variables and obtaining the objective function from the Monte Carlo simulation model.

- Starting recursively from the last decision node, the expected future value of holding the option is compared with the immediate value of exercising the option. The expected future value is conditional on the resolution of the uncertainties until that time. This expected future value is equivalent to the value of continuation.

- Estimating the continuation value by regressing the simulated future outcomes onto basis functions of the state variables at the time of the decision. This relates the continuation value to the state variables at a specific time.

- Determining the optimal policy by choosing the option that achieves the highest value given the known information.

Jafarizadeh and Bratvold (2009) provide a detailed, step-by-step description of the LSM method.

\section{Monte Carlo Simulation}

The first component of the method is to perform a Monte Carlo simulation of the surfactant flood for all options considered. That implies running the reservoir simulator and obtaining the NPV for surfactant initiation at $y_{n}=(4,5,6,7)$ encompassing the option scenario. A total of $10^{3}$ paths were simulated to produce a statistical base. For each path, a unique realization of $S_{\text {orc }}, D_{s}, P r_{o}, P r_{s}$, $P r_{w i}$, and $P r_{w p}$ is obtained. The stochastic processes for each of these parameters are discussed below.

Surfactant-Flooding Modeling. The effects of uncertainty on EOR processes have been well documented over the years. Although it is considered the EOR process with the highest recovery potential, surfactant flooding represents a small fraction of commercially successful EOR projects. This is mainly because of uncertainty (Lake 1989, Thomas 2006). There are many sources of uncertainty in surfactant flooding such as heterogeneity, surfactant adsorption, brine salinity, and ROS to chemical flooding. The effect of reservoir heterogeneity on chemical flooding was recognized as a dominant factor in earlier studies that influenced volumetric sweep (Kossack and Bilhartz 1976; Brown and Smith 1984; Hankins and Harwell 1996; Cheng et al. 2012). ROS to chemical flooding is considered to be the most significant factor affecting recovery efficiency of the EOR process, whereas surfactant adsorption was also considered to have a dominant adverse effect (Brown and Smith 1984; Dang et al. 2011; Solairaj et al. 2012). Salinity effects were studied extensively and found to influence recovery significantly (Gerbacia 1978; Pope et al. 1979; Hirasaki et al. 2011; Puerto et al. 2012; Lohne and Fjelde 2012).

The ROS to chemical flooding, $S_{\text {orc }}$, and surfactant adsorption, $D_{s}$, were assumed to be the technical uncertainties in this study, and they are assumed to follow stochastic processes. No correlation was assumed between these two parameters. Schlumberger's ECLIPSE (ECLIPSE 2010) was used as the reservoir simulator, and code was written in MATLAB that performed the stochastic modeling and began the reservoir simulations. Two reservoir models were used: a homogeneous 3D model and a heterogeneous 2D model. These models were chosen because they are standard stylized models that can be easily reproduced and are used to demonstrate the applicability of the method. Tables A-1 through A-6 show the reservoir-model data and constraints used. A surfac- tant-slug size of $10 \%$ pore volume (PV) and a concentration of 10 $\mathrm{lbm} / \mathrm{bbl}$ injected (3 wt\%) were assumed. The surfactant-slug variables are held constant for all runs. It is important to state that because we did not aim to solve a specific reservoir problem here, we used standard distributions for the uncertain parameters to demonstrate the method suggested in this paper. The effect of the evolution of these parameters is then manifested in the production profiles that were determined by simulation.

The reservoir state variables are $S_{\text {orc }}$ (fraction) and $D_{s}(\mathrm{lbm} /$ lbm rock), which will initially follow a stochastic process of uniform $\sim U(0,0.20)$ and $\log$-uniform $\sim \log U(-6,-3)$ distributions, respectively. These distributions were chosen because they are simple standard distribution models that capture a certain range for each of the technical state variables. Other common distributions could also be applied (e.g., normal or beta). There will be a distinct realization of both for each path. Because we assume that our knowledge (information) set evolves through time, the state variables are also assumed to evolve through time. This is assumed to follow a simple stochastic process defined as a discrete-time continuous-state random-walk-with-jump process. It can be considered a Markov process because the future value of the variable will depend only on its current value, not on the realized values in the past. Realizations are found at discrete points in time that coincide with the decision nodes (i.e., at 4, 5, 6, and 7). The stochastic process for $S_{\text {orc }}$ can be presented as

$$
S_{\text {orc }}=x_{t} \quad S_{\text {orc }}=x_{t}=x_{t-1}+\rho \varepsilon_{t},
$$

where $x_{t}$ is the state variable $\left(S_{\text {orc }}\right)$ and $\rho$ is the jump direction, $\rho \in(-1,0,1), P(\rho=-1)=P(\rho=1)=P(\rho=0)=\frac{1}{3}$.

$\varepsilon_{t}$ is a random variable with distribution

$$
\varepsilon_{t} \sim U\left[\left(1-q_{t}\right) x_{t-1},\left(1+q_{t}\right) x_{t-1}\right] .
$$

$q_{t}$ is the range adjustment (Table $\mathbf{1}$ ).

The stochastic process for $D_{s}$ follows a similar process:

$$
\begin{aligned}
D_{s_{t}}=10^{x_{t}}, \text { where } x_{t}=x_{t-1}+\rho \varepsilon_{t} \\
\quad \text { and } \varepsilon_{t} \sim U\left[\left(1-q_{D t}\right) x_{t-1},\left(1+q_{D t}\right) x_{t-1}\right] .
\end{aligned}
$$

The range adjustment values are stylized values that are used in this study to demonstrate the method. The realization of the technical state variable is kept constant between decision nodes as the simulation proceeds. This process is intended to mimic our increasing state of knowledge of the value of these parameters with time. See Fig. 1 and Fig. 2 for sample realizations of the first five paths for the homogeneous (HMG) and heterogeneous (HTG) models.

Homogeneous Model. The homogeneous model was designed as a $10 \times 10 \times 10$ grid and a quarter-five-spot pattern with a total area of 5 acres and a reservoir thickness of $25 \mathrm{ft}$. Sample production profiles of the first five paths (realizations) with surfactant flooding initiated at the start of the fourth year and of the first path for all injection scenarios are presented in Fig. 3. It is clear that different realizations of $S_{\text {orc }}$ and $D_{s}$ will affect the recovery factors achieved from the process. Also, it is clear that beginning surfactant flooding at different times will achieve different recovery factors.

Heterogeneous Model. The model is derived from Layer 1 of the SPE10 model (Christie and Blunt 2001) adapted to a 5-acre quarter-five-spot pattern with a thickness of $25 \mathrm{ft}$. The fluid data used were those of the homogeneous model. Heterogeneity is assumed to be an exogenous source of uncertainty that evolves through time with our evolving state of information. We assumed that the fine $(60 \times 220)$ model is realized at the last decision node $(y=7)$ onward. Going back in time, the model is scaled up at each decision node $(y=6,5$, and 4$)$ until the first decision node at $y=4$, for which the realization is kept constant until the start of the field life.

Fig. 4 shows the evolution of heterogeneity with time in terms of the natural logarithm of permeability in millidarcies. From left to right, the permeability fields evolve and take more details. In designing an artificial increase in detail, we implemented a 

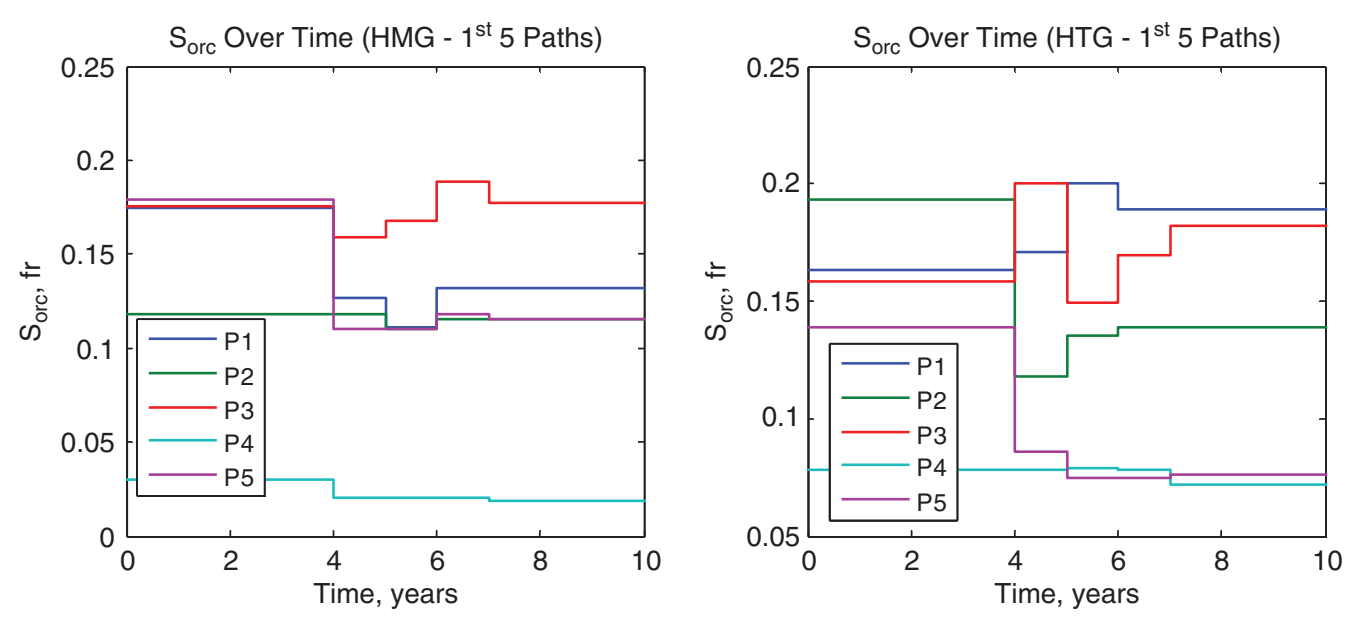

Fig. 1-ROS-to-chemical-flooding variation with time for the homogeneous and heterogeneous models.
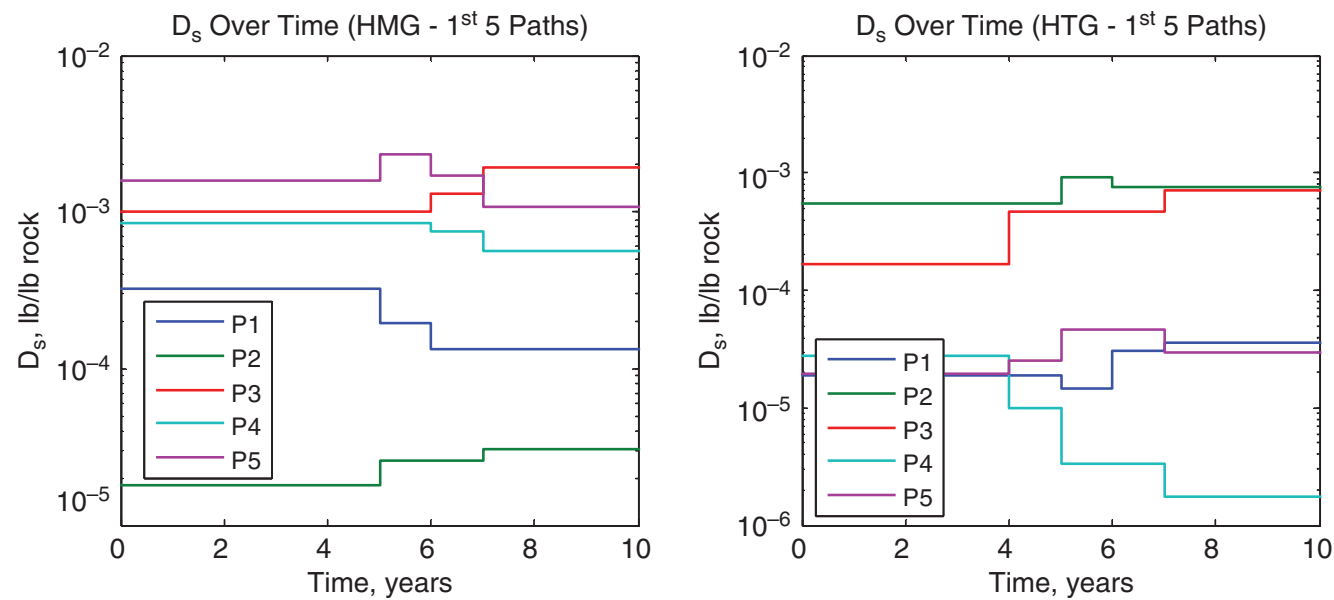

Fig. 2-Surfactant-adsorption variation with time for the homogeneous and heterogeneous models.

reverse upscaling procedure. The original geologically detailed model represents the most evolved state of permeability (at farright panel of Fig. 4). For the next step, an absolute upscaling of permeability is performed on a $60 \times 220$ grid layout to coarsen the model to a $30 \times 110$ grid. Then, a refining stage maps the new layout back to a $60 \times 220$ grid. This stage is simply performed by replacing each cell with four cells of similar values for permeability. This procedure was continued to $15 \times 55$ and $6 \times 22$ grids. The upscaling procedure used here is the pressure-solver method

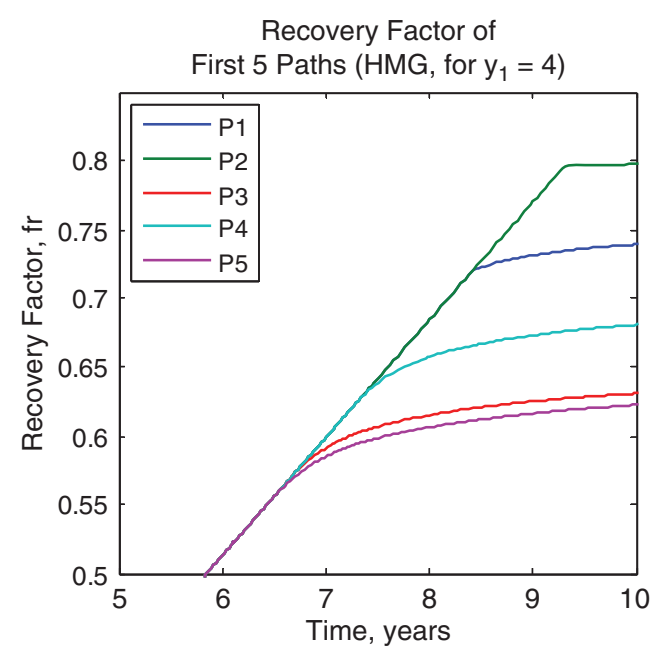

(Warren and Price 1961; Begg et al. 1989). We use a simple procedure that calculates simulation gridblock equivalent permeabilities with only diagonal elements in the tensor. This is achieved by inverting a fine pressure solution calculated locally over the domain of each simulation coarse gridblock. For Layer 1 of the SPE10 model, the simulation results of the upscaled model by the directional pressure-solver method are of good quality compared with the fine-scale simulations. This is because of the smoothness of the variation of the permeability field for this layer.

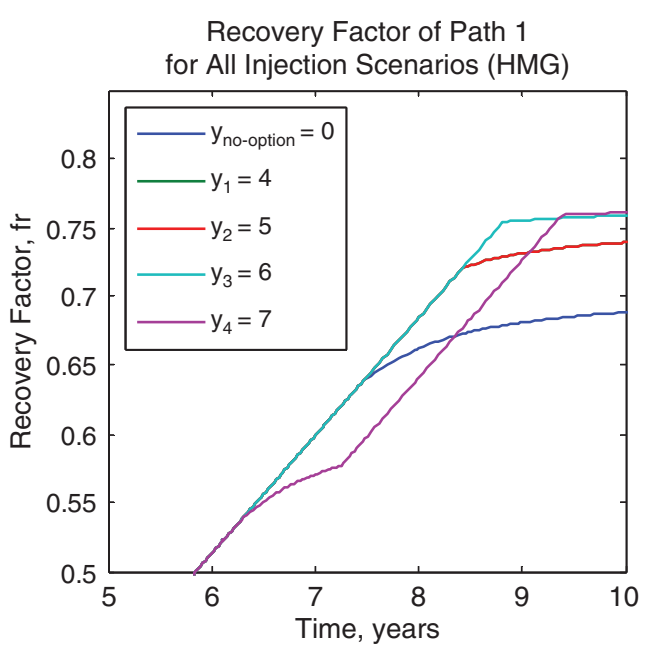

Fig. 3-Production profiles for homogeneous model. 

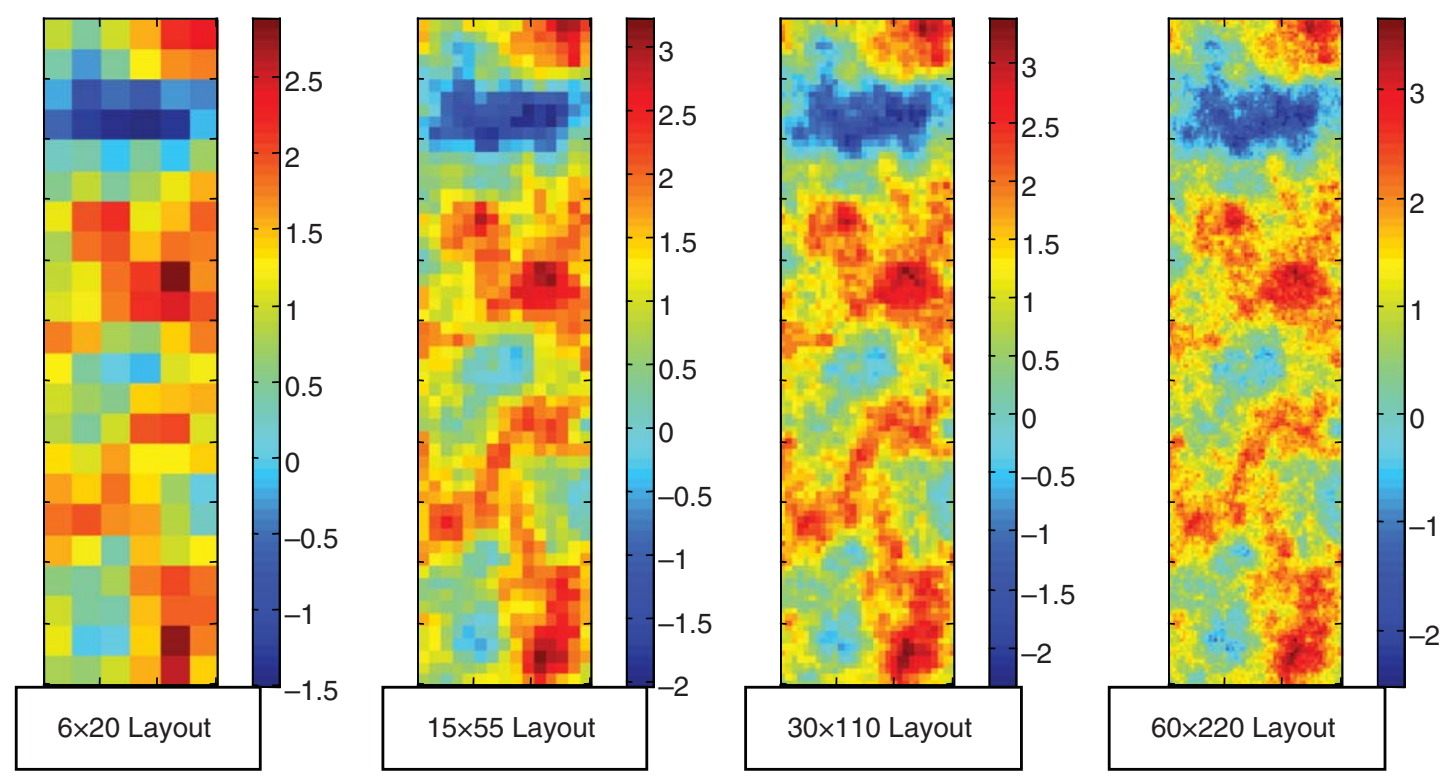

Fig. 4-Heterogeneity variation with time, with the far left showing the coarsest realization and the far right showing the finest realization of the permeability field.

For such layers, the results of the application of most common upscaling methods (for example, harmonic-arithmetic mean and renormalization method) are essentially identical to those of the pressure-solver method and are equally satisfactory.

On the other hand, as shown in a comparison study of several upscaling methods in Babaei and King (2012), the upscaling errors of the directional pressure-solver method for more-heterogeneous layers can be significantly higher. In such circumstances, other advanced upscaling methods are required to prevent the upscaling error from undermining the reliability of the upscaled models. In other words, we will have additional scale-related uncertainties in use of the models at different stages of upscaling or reverse upscaling. One thing that we want here is to have correctly increasing details in reservoir heterogeneity, or a fine-scale model that is matching with the coarse-scale model; hence we limited the example to a model with heterogeneity pattern like that of Layer 1 of the SPE10 model. Fig. 5 shows sample production profiles for the first five paths (realizations) for surfactant injection at the beginning of the fourth year and for Path 1 for surfactant injection under all scenarios. From both figures, the difference in recovery factors is more pronounced-as is expectedcompared with the homogeneous model.

Recovery Factor of First 5 Paths (HTG, for $\left.\mathrm{y}_{1}=4\right)$

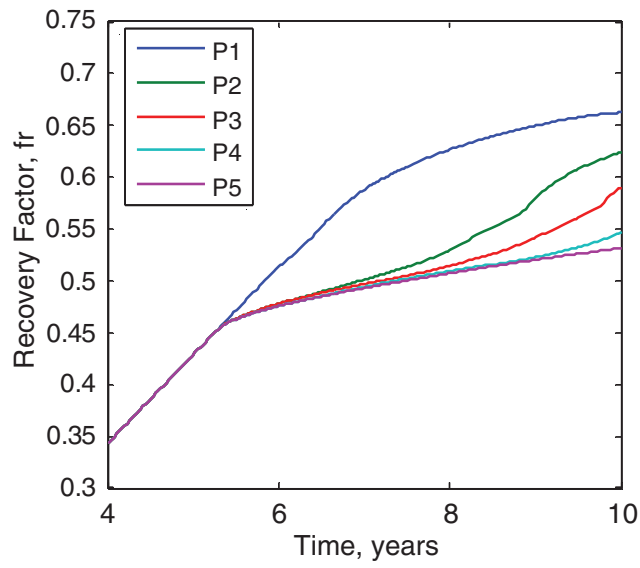

Economic Modeling. The objective function used in the decision criteria is the NPV. The economic model will be based on calculating net revenue as

$$
\begin{aligned}
\text { Net Revenue }= & \sum[\text { Oil Revenue } \\
& -(\text { Cost of Injected Water } \\
& + \text { Cost of Produced Water } \\
& + \text { Cost of Surfactant })] .
\end{aligned}
$$

Risk-neutral valuation is used when the oil-price stochastic model is adjusted for risk with a risk premium of 3\% [risk premium value is obtained from the oil-price model in Willigers and Bratvold (2009)]. This approach is more comprehensive in analyzing cash flows because it adjusts every component of the cash flow for risk and then discounts for time (at the risk-free rate), whereas traditionally a constant discount factor (including both time and risk) was used that could generate biased results caused by the varying risk patterns of the component cash flows (Jafarizadeh and Bratvold 2009). Cash flows are then discounted with the risk-free rate, $r$, which is assumed to be constant over time and equal to $6 \%$. Continuous discounting is used.

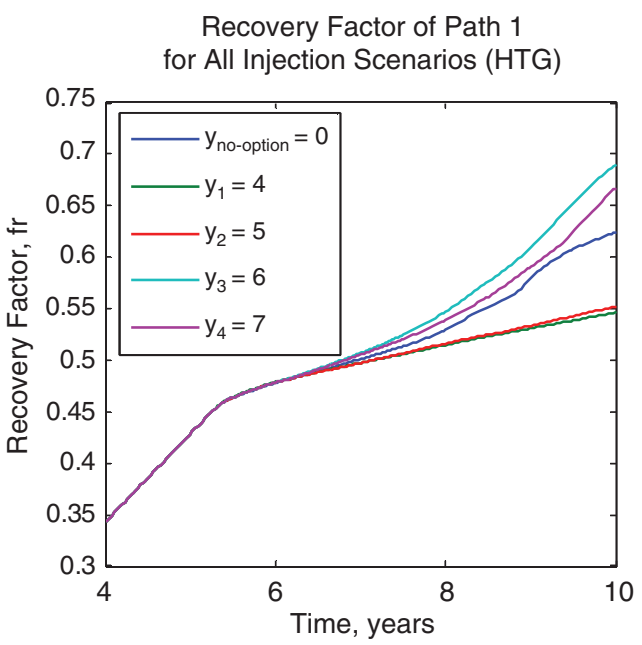

Fig. 5-Production profiles for heterogeneous model. 
TABLE 2-ORNSTEIN-UHLENBECK PROCESS PARAMETERS

\begin{tabular}{|lc|}
\hline Process Parameters & Values \\
\hline$t$, months & 120 \\
$d t$ & 1 \\
$\lambda$ & 0.0012 \\
$\mu, \mathrm{USD} / \mathrm{bbl}$ & 66.67 \\
$\sigma$ & 1.81 \\
$\delta$ & 0.03 \\
$\operatorname{Pr}_{o} S_{0}, \mathrm{USD} / \mathrm{bbl}$ & 90 \\
\hline
\end{tabular}

The $\lambda, \mu$, and $\sigma$ parameters were obtained using the least-squares method to estimate parameters of an observed Ornstein-Uhlenbeck process (Smith 2010). NYMEX futures prices from April 1983 to July 2012 were used as the basis of the estimation (EIA 2012). $t$ and $d t$ are based on a simulation period of 120 months (10 years) discretized into monthly timesteps.

There are four economic state variables: the oil price $\left(P r_{o}\right)$, the surfactant cost $\left(P r_{s}\right)$, cost of water injection $\left(P r_{w i}\right)$, and the cost of produced water $\left(P r_{w p}\right)$. $P r_{o}(\mathrm{USD} / \mathrm{STB})$ and $P r_{s}$ (USD/STB surfactant) will be modeled independently, whereas $P r_{w i}$ (USD/STB) and $P r_{w p}$ (USD/STB) are correlated [following Jafarizadeh and Bratvold (2009)] with $\operatorname{Pr}_{o}$. $P r_{o}$ is modeled as a risk-neutral Ornstein-Uhlenbeck process (Uhlenbeck and Ornstein 1930), which is a mean-reverting stochastic process:

$$
d P r_{o} S=\lambda\left[\left(\mu-\frac{\delta}{\lambda}\right)-P r_{o} S\right] d t+\sigma d W_{t}
$$

where $W_{t}$ is a Brownian motion, $d W_{t} \sim N(0, \sqrt{d t}) ; \lambda$ is the measure of the speed of mean reversion; $\mu$ is the long-term mean that the process reverts to; $\sigma$ is the measure of the process volatility; and $\delta$ is the risk premium.

To simulate the oil price with Eq. 5, it is necessary to discretize it with respect to time. The following provides an exact discrete-time expression as

$$
P r_{o} S_{t}=e^{-l \Delta t} P r_{o} S_{t-1}+\left(1-e^{-l \Delta t}\right)\left(\mu-\frac{\delta}{l}\right)+\sigma \sqrt{\frac{\left(1-e^{-2 l \Delta t}\right)}{2 l}} d W_{t}
$$

This discrete-time version of the process allows an exact discretization in that accuracy is not reduced if a larger timestep is used (Dias 2004). A more detailed discussion of why the previously discussed discretization of a mean-reverting process is chosen can be found in Dixit and Pindyck (1994). Mean-reverting stochastic processes are well suited for modeling commodity prices because they reflect the economic argument that when prices are too high, supply will increase and demand will reduce. When prices become

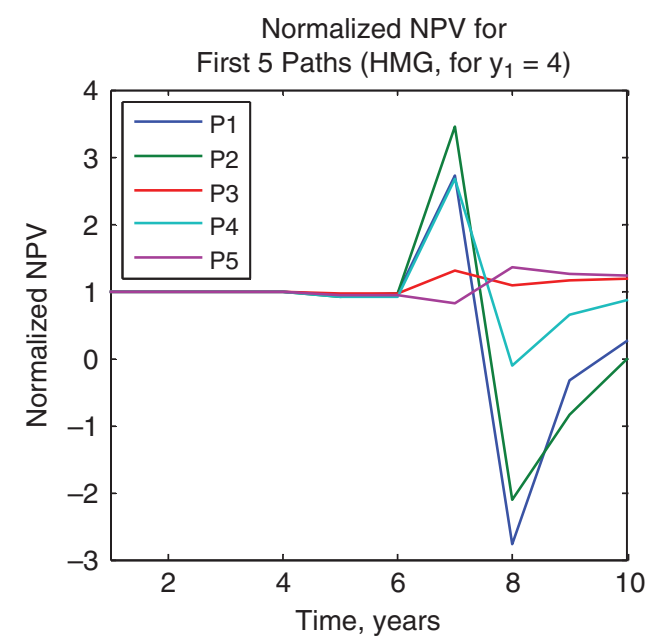

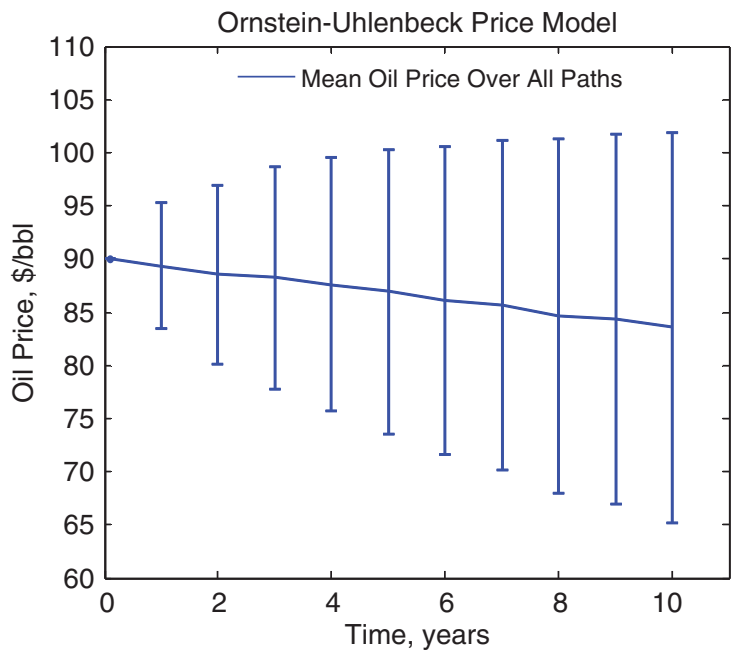

Fig. 6-Ornstein-Uhlenbeck price model, showing the mean of 1,000 paths and the corresponding standard deviation.

too low, the opposite effect occurs, pushing prices toward the longterm mean (Smith 2010). The Ornstein-Uhlenbeck process parameters are shown in Table 2. Fig. 6 shows the price model.

The surfactant price $\left(\mathrm{Pr}_{s}\right.$; price range derived from Anderson et al. 2006; Thomas 2006; Wyatt et al. 2008) is modeled with a uniform distribution, $\sim U(1,3)$. A unique realization at each timestep is sampled from the distribution. For water-injection cost $\left(P r_{w i}\right)$ and water-production cost $\left(P r_{w p}\right)$, we applied a cost multiplier factor of 0.10 to the oil price $\left(P r_{o}\right)$.

Figs. 7 and 8 show sample normalized NPV charts for the same technical sample realizations presented in the preceding subsection. This is performed for all injection scenarios for the first path (realization) and for the first five paths under a constantinjection scenario of $y_{1}=4$. These figures are normalized onto the basis of the NPVs calculated for regular waterflood with the same stochastic prices generated from the mean-reverting process discussed previously for each path. It is important to note that we did not include any capital-expenditure considerations in the economic model because our main aim here is to introduce the method; we assumed that existing equipment can be used and adapted for surfactant flooding at negligible cost, although including capital costs would be a natural progression in enhancing the economic model.

After completing the forward Monte Carlo simulation of the surfactant flood, the LSM algorithm is then implemented. The LSM algorithm is discussed in more detail in the next section. Fig. 9 shows a flow chart summarizing the MATLAB algorithm written for this study.

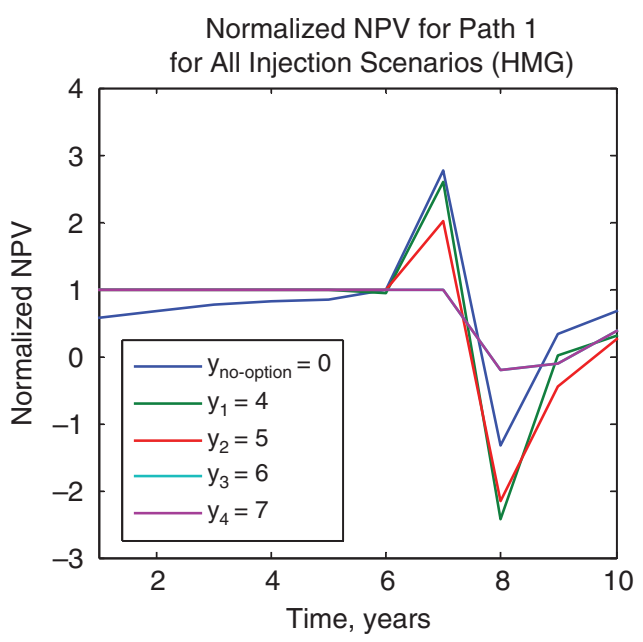

Fig. 7-Normalized NPV for the homogeneous model. 
Normalized NPV for

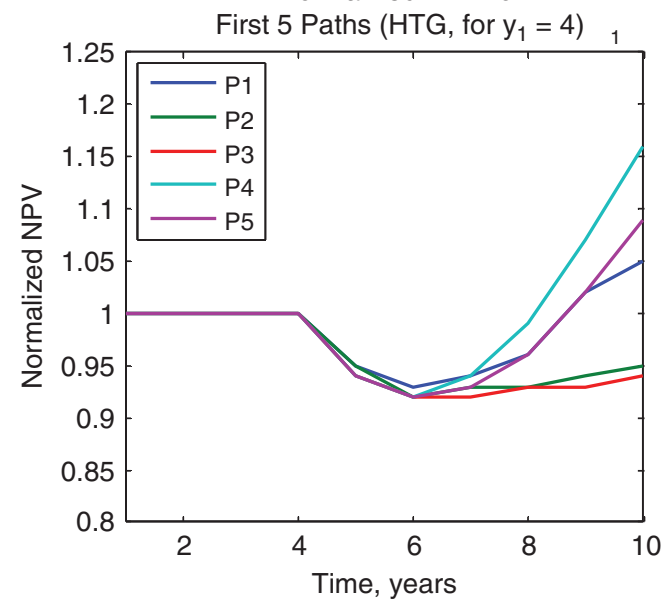

Normalized NPV for Path 1

for All Scenarios (HTG)

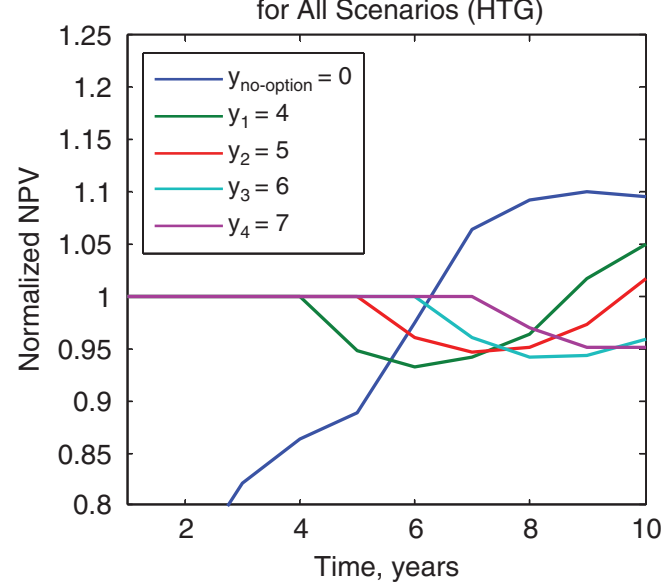

Fig. 8-Normalized NPV for the heterogeneous model.

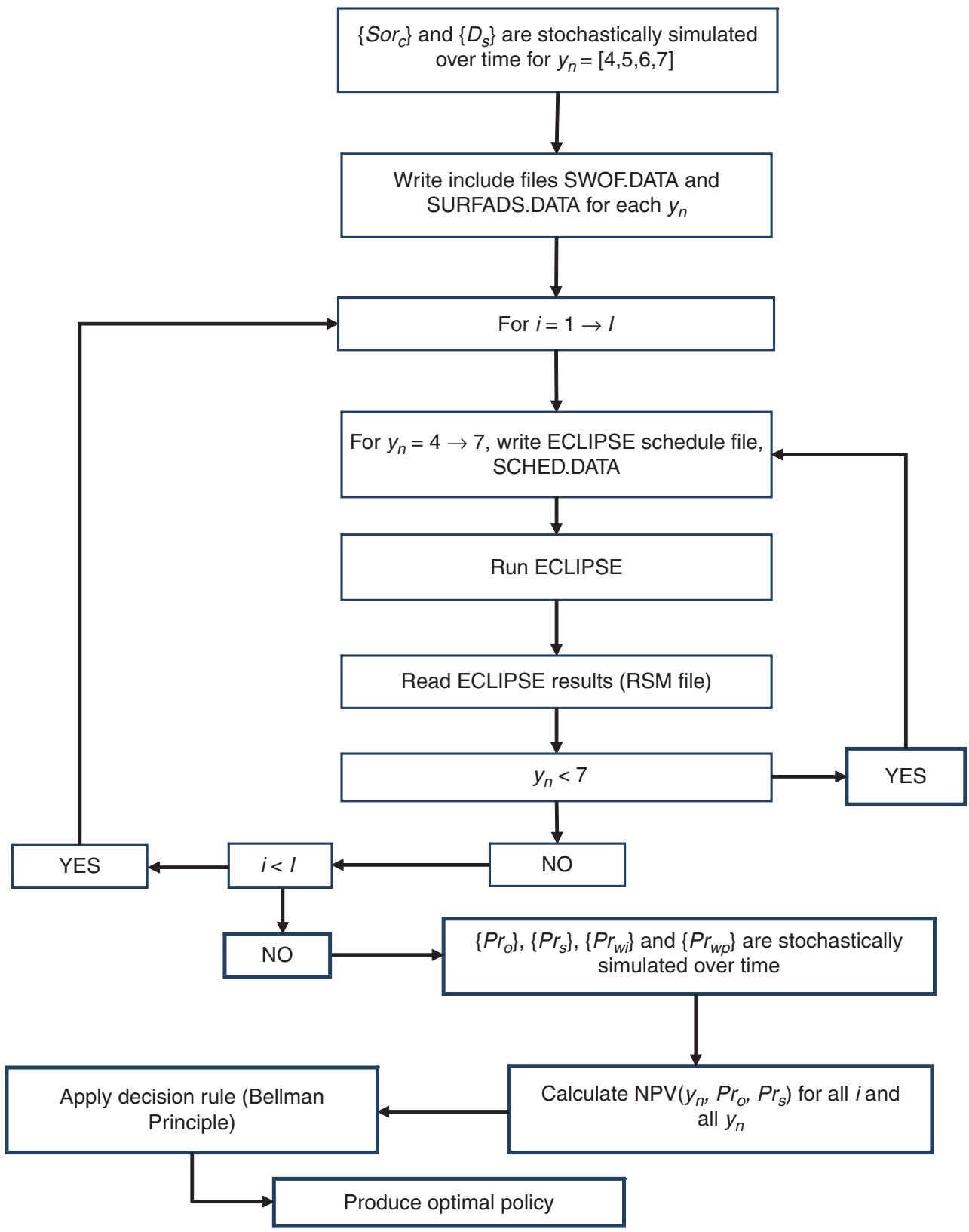

Fig. 9-Algorithm flow chart illustrating the steps followed in the MATLAB code. 


\section{LSM Algorithm}

We define a variable $t$ to be the optimal time to begin the surfactant flood (i.e., exercise the option) and $t(x)$ to be the optimal exercise time for a certain realization path. $(x)$ is defined as one realization of the evolution of the state variables over a time horizon of 10 years and $x \in X . X$ is the total number of stochastic simulations. The state variables used in the algorithm are the economic uncertainties: the oil price $\left(P r_{o}\right)$ and the surfactant price $\left(P r_{s}\right)$. The technical uncertainties are separate from the economic uncertainties, and their influence is manifested in the production profile of the reservoir model. The other economic parameters are correlated with $\mathrm{Pr}_{o}$ and are incorporated in the NPV calculation. As mentioned previously, there will be a distinct realization of the state variables for each path, $(x)$. Because we assume that our knowledge (information) set evolves through time, the state variables are assumed to evolve through time. Therefore $P r_{o}$, for instance, will be depicted with another subscript, $y_{n}$, which will illustrate the time dependencies of the state variables. Here $y_{n}$ is the decision time that is an element of the set of decision times, $Y=\left[y_{1}, \ldots, y_{n}\right]$; consequently the optimal exercise time, $t(x) \in Y$. Hence, $\operatorname{Pr}_{o, y_{n}}(x)$ is the oil price at the decision node time $y_{n}$ along the $x$ th simulated path. Assume we have the payoff function $N P V\left[y_{n}, \operatorname{Pr}_{o, y_{n}}(x), \operatorname{Pr}_{s, y_{n}}(x)\right]$, which is the NPV of the process (NPV at the end of the 10-year simulation interval) when we exercise the option to begin the surfactant flood, dependent on the realization of the state variables, at time $y_{n}$. This function will be defined as the immediate option exercise value.

$O N P V$ is defined as the optimal value function, which is the value of the optimal NPV at the end of the simulated field life dependent on the exercise of the optimal surfactant initiation time (i.e., the option). Then, we have

$$
\begin{aligned}
t(x) & =y_{n}, \text { if } \operatorname{ONPV}\left[y, \operatorname{Pr}_{o, y}(x), \operatorname{Pr}_{s, y}(x)\right] \\
& =N P V\left[y_{n}, \operatorname{Pr}_{o, y_{n}}(x), \operatorname{Pr}_{s, y_{n}}(x)\right] .
\end{aligned}
$$

Because $t(x)$ is the optimal surfactant initiation time per path $(x)$, it is assumed to follow this condition:

$$
\begin{aligned}
t & =\inf \left[y \mid \operatorname{ONPV}\left(y, \operatorname{Pr}_{o, y}, \operatorname{Pr}_{s, y}\right)\right. \\
& \left.=\operatorname{NPV}\left(y_{n}, \operatorname{Pr}_{o, y_{n}}, \operatorname{Pr}_{s, y_{n}}\right)\right], \quad \cdots
\end{aligned}
$$

which is the first time (i.e., decision node) when the option value equals the payoff achieved from exercising the option.

At this step, $\operatorname{ONPV}\left(y, \operatorname{Pr}_{o, y}, \operatorname{Pr}_{s, y}\right)$ is not available. However, it can be determined with Bellman's formula:

$$
\begin{aligned}
& O N P V\left[y, \operatorname{Pr}_{o, y}(x), \operatorname{Pr}_{s, y}(x)\right]=\max \left(N P V\left(y_{n}, \operatorname{Pr}_{o, y_{n}}, \operatorname{Pr}_{s, y_{n}}\right),\right. \\
& \left.e^{-r\left(y_{n+1}-y_{n}\right)} E_{y_{n}}^{*}\left\{\operatorname{ONPV}\left[y_{n+1}, \operatorname{Pr}_{o, y_{n+1}}(x), \operatorname{Pr}_{s, y_{n+1}}(x)\right]\right\}\right),
\end{aligned}
$$

where $r$ is the discount factor and $E_{y_{n}}^{*}$ is the expectation function. We define the continuation function as

$$
\begin{aligned}
& \mathrm{F}\left[y_{n}, \operatorname{Pr}_{o, y_{n}}(x), \operatorname{Pr}_{s, y_{n}}(x)\right]=e^{-r\left(y_{n+1}-y_{n}\right)} \\
& \times E_{y_{n}}^{*}\left\{\operatorname{ONPV}\left[y_{n+1}, \operatorname{Pr}_{o, y_{n+1}}(x), \operatorname{Pr}_{s, y_{n+1}}(x)\right] \mid \vartheta_{y_{n}}\right\}, \ldots \ldots
\end{aligned}
$$

where $\vartheta_{y_{n}}$ is the information set $\left[\operatorname{Pr}_{o, y_{n}}(x)\right.$ and $\left.\operatorname{Pr}_{s, y_{n}}(x)\right]$ at time $y_{n}$. Thus, the decision rule at time $y_{n}$ along the $x$ th path is

$$
\begin{aligned}
& \text { if } \mathrm{F}\left[y_{n}, \operatorname{Pr}_{o, y_{n}}(x), \operatorname{Pr}_{s, y_{n}}(x)\right] \leq N P V\left[y_{n}, \operatorname{Pr}_{o, y_{n}}(x), \operatorname{Pr}_{s, y_{n}}(x)\right], \\
& \text { then } t(x)=y_{n} . \ldots \ldots \ldots \ldots \ldots \ldots \ldots \ldots \ldots \ldots \ldots \ldots \ldots
\end{aligned}
$$

To determine the optimal policy, the decision rule is applied recursively from $y_{n}=y_{N}$ back to $y_{n}=y_{1}$. When the algorithm reaches $y_{n}=y_{1}$ and all the optimal policies (surfactant initiation times) have been determined along all paths, the value of the option is determined by calculating the average of the optimal values of the objective function (simulated NPV in this case) for all paths:

$$
\begin{aligned}
& O P N V\left[y_{1}, \operatorname{Pr}_{o}(x), \operatorname{Pr}_{s}(x)\right] \\
& =\frac{1}{X} \sum_{x=1}^{x} e^{-r t(x)} N P V\left[t(x), \operatorname{Pr}_{o, t(x)}(x), \operatorname{Pr}_{s, t(x)}(x)\right] .
\end{aligned}
$$

Averaging is justified because the value of an American option (the option considered in this study is analogous to an American option, which can be exercised during any time until it expires) can be represented by the Snell envelope (Bensoussan 1984; Karatzas 1988). This implies that the value of an American option equals the maximized value of the discounted cash flows from the option. The maximum is taken over all stopping times. The main objective of the LSM algorithm is to provide a pathwise approximation to the optimal stopping rule that maximizes the value of the American option.

To apply the decision rule, the continuation function, $\mathrm{F}$, needs to be determined at $\left[y, \operatorname{Pr}_{\mathrm{o}, t(x)}(x), \operatorname{Pr}_{s, t(x)}(x)\right]$. The algorithm assumes that the continuation value is the expectation, dependent on the available information at the time of the decision, of the future optimal values. Thus, the continuation value is the present value of the future expected payoffs:

$$
\begin{aligned}
& \mathrm{F}\left[y_{n}, \operatorname{Pr}_{o, y_{n}}(x), \operatorname{Pr}_{s, y_{n}}(x)\right] \\
& =E_{y_{n}}^{*}\left\{\sum_{i=n+1}^{N} e^{-r\left(y_{i}-y_{n}\right)} N P V\left[y_{n}, \operatorname{Pr}_{o, y_{n}}(x), \operatorname{Pr}_{s, y_{n}}(x)\right]\right\} .
\end{aligned}
$$

The continuation value can be represented as follows because $\mathrm{F}$ is an element of a linear vector space, belonging to the Hilbert space:

$$
\mathrm{F}\left[y, P r_{o, y}(x), P r_{s, y}(x)\right]=\sum_{j=1}^{\infty} \varnothing_{j}(y) L_{j}\left(y, P r_{o, y}, P r_{s, y}\right) . \ldots .
$$

$L_{j}$ is the $j$ th element in the orthonormal basis and $y \in$ $\left[y_{1}, \ldots, y_{n}\right] . L_{j}\left(y, P r_{o, y}, P r_{s, y}\right)$ can be powers of the state variables, Laguerre or Hermite polynomials (Longstaff and Schwartz 2001). Here, for simplicity, it is assumed to be a polynomial of the second order (without cross products) in the form $\alpha P r_{o, y_{n}}^{2}(x)+$ $\beta \operatorname{Pr}_{o, y_{n}}(x)+\rho P r_{s, y_{n}}^{2}(x)+\pi P r_{s, y_{n}}(x)+C$, where $\alpha, \beta, \rho$, and $\pi$ are coefficients and $C$ is a constant. For simple decision problems, the choice of basis functions can be straightforward and different polynomials can produce similar results; however, for more-complex decision problems, the type of basis functions can affect the results obtained (Moreno and Navas 2003). (Different polynomial families were used as basis functions up to the third order. These included Chebyshev, Hermite, Laguerre, and Legendre polynomials with cross products. The results obtained for the decision presented in this study were similar to those obtained with the simple second-order polynomial, presented previously.) Therefore, it is important to perform a sensitivity analysis of the type of basis functions used in the algorithm.

If $J<\infty$ elements in the basis are used to determine $\mathrm{F}$, then we obtain an approximation of the continuation value with

$$
\mathrm{F}^{J}\left(y, \operatorname{Pr}_{o, y}, \operatorname{Pr}_{s, y}\right)=\sum_{j=1}^{J} \varnothing_{j}(y) L_{j}\left(y, \operatorname{Pr}_{o, y}, P r_{s, y}\right) . \quad \ldots \ldots
$$

It is assumed that the unknown functional form of the continuation equation can be represented as a linear combination of a countable set of the information set (state variables) measurable basis functions. The continuation function is approximated (the continuation value approximation is truncated to $J=4$ for the examples in this study) with the first $J<\infty$ basis functions (Longstaff and Schwartz 2001). $\varnothing_{j}(y)$ can be represented by a linear least-squares regression of $\mathrm{F}^{J}\left(y, P r_{o, y}, P r_{s, y}\right)$ onto the basis $\left[L_{j}\left(y, P r_{o, y}, P r_{s, y}\right)\right]$ :

$$
\begin{aligned}
& \left\{\hat{\varnothing}\left(y_{n}\right)\right\}_{j=1}^{J}=\operatorname{argmin}_{\left\{\varnothing_{j}\right\}_{j=1}^{J}} \| \sum_{j=1}^{J} \varnothing_{j}\left(y_{n}\right) L_{j}\left(y_{n}, P r_{o, y_{n}}, P r_{s, y_{n}}\right) \\
& -\sum_{i=n+1}^{N} e^{-r\left(y_{i}-y_{n}\right)} \operatorname{NPV}\left(y, P r_{o, y}, P r_{s, y}\right) \|^{2} \cdot \ldots \ldots \ldots
\end{aligned}
$$



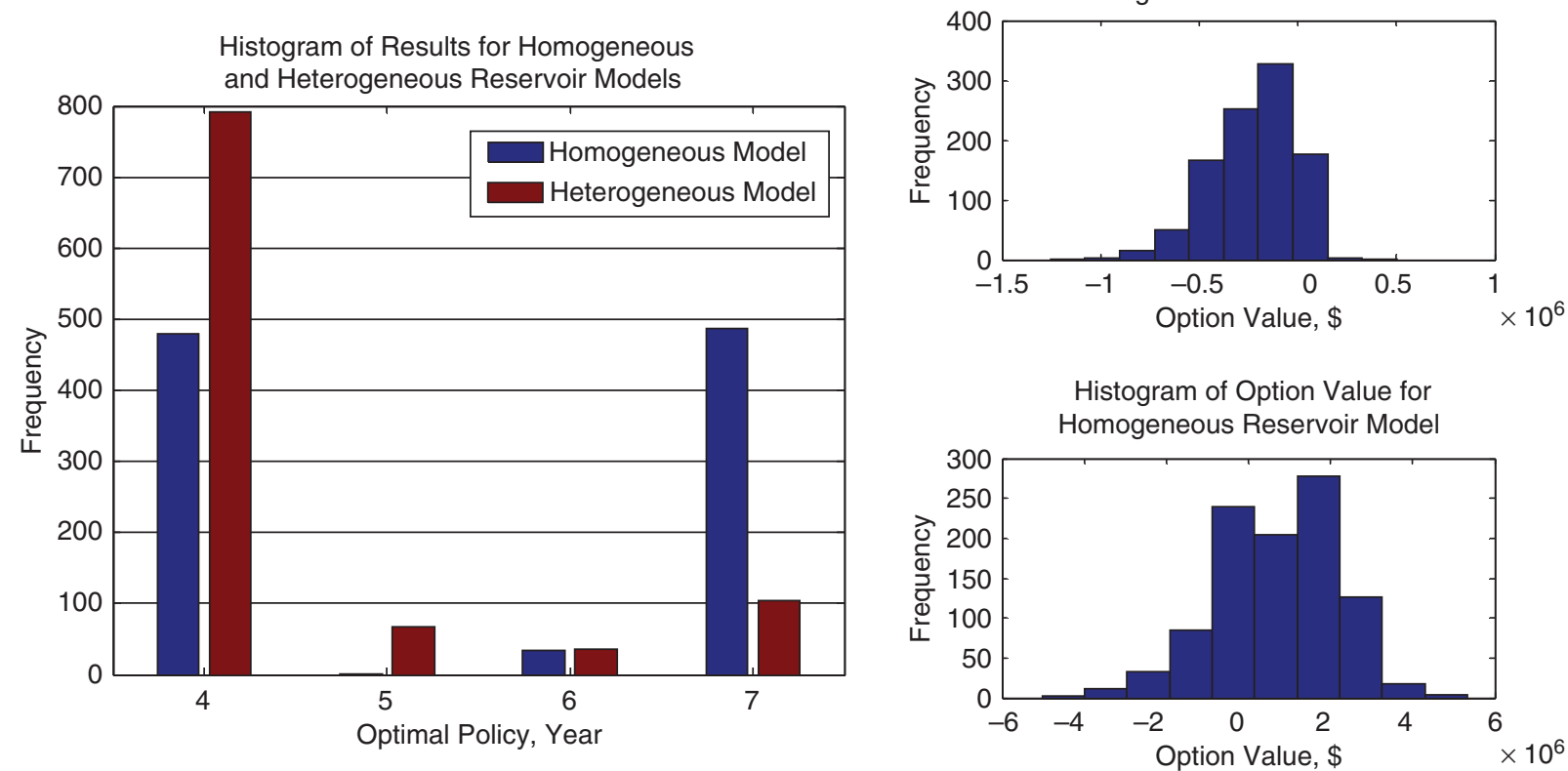

Fig. 10-Histograms of optimal policy and option value for both the homogeneous and heterogeneous reservoir models. Option value is determined as the difference between the average value of the option scenario (i.e., injection at $y_{n}=4,5,6$, or 7 ) over all realizations and that of the average value of the static scenario over all realizations.

Because the values of the basis functions are independently and identically distributed across paths, weak assumptions about the existence of moments enable the use of Theorem 3.5 of White (1984) to illustrate that the resultant fitted value of the regression converges in mean square and in probability to the approximation function as the number of paths goes to infinity. Theorem 1.2.1 (Amemiya 1985) implies that the fitted values of the regression are the best linear unbiased estimator of the approximation function on the basis of a mean-squared metric (Longstaff and Schwartz 2001).

Therefore, the estimated continuation value estimate is

$$
\hat{\Phi}^{J}\left(y_{n}, \operatorname{Pr}_{o, y_{n}}, \operatorname{Pr}_{s, y_{n}}\right)=\sum_{j=1}^{J} \hat{\varnothing}_{j}\left(y_{n}\right) L_{j}\left(y, \operatorname{Pr}_{o, y_{n}}, \operatorname{Pr}_{s, y_{n}}\right) \text {. }
$$

In summary, the algorithm was presented as a simple and efficient method for option valuation. Its main assertion is that if at a certain time the option is still available, then the continuation value is the expectation (conditional on the information available at that time) of the future optimal payoffs from the option (Gamba 2002). The main aspect of the LSM approach is the regression of the ex post realized payoffs from continuation on functions of values of the state variables. The regression provides a fitted value that is a direct estimate of the conditional expectation function (Longstaff and Schwartz 2001). The LSM method is actually a form of approximate dynamic programming (Powell 2011).

Accuracy of the estimated values of the option can be enhanced by increasing the number of timesteps, number of simulated paths, and number of basis functions (Gamba 2002). The choice of basis functions is clear in the case of a simple option; all that is needed is to select the degree of a polynomial on the state variable. Different polynomials produce similar results; this is so because any polynomial can be expressed as a linear combination of others (Moreno and Navas 2003). For some cases, it might be more efficient to use other regression techniques such as weighted least squares, generalized least squares, and generalized method of moments in estimating the conditional expectation function (Longstaff and Schwartz 2001).

\section{Results and Discussion}

A simple decision was analyzed in this study with regard to a surfactant-flooding process. Uncertainty in technical and economic parameters was considered along with the uncertainty in the time series of these variables. With the LSM method, it was possible to perform this analysis and produce an optimal policy. This policy represents the influence of uncertainty in the time series of the relevant input parameters, which provides a framework to either capitalize on upside opportunities or mitigate downside circumstances. The method also determines the value of flexibility of the decision analyzed, in which the value of flexibility is the value of splitting decisions into multiple decisions over time with the opportunity to learn between decisions and the option to respond to that learning (Bratvold and Begg 2010).

Two permeability-field models were used. The first model was a 3D homogeneous model that represents the simplest realization of a reservoir with respect to permeability. The second model was a 2D heterogeneous model that is derived from the first layer of the SPE10 model adapted to a 5-acre quarter-five-spot pattern. The homogeneous model assumes the permeability field remains constant, whereas the heterogeneous model assumes its permeability field evolves with time. The technical state variables were assumed to evolve through time after being initially sampled with predefined distributions. Their evolution through time was assumed to be a Markov process following a discrete-time continuous-state random-walk-with-jump process. The economic model was rooted in risk-neutral valuation with a risk-adjusted stochastic model for the oil price. Capital expenditures were assumed to be nonexistent because it was deemed that existing surface equipment could be adapted to surfactant flooding at negligible cost.

The results (Fig. 10) obtained for the homogeneous model show that the optimal policy was for surfactant injection for nearly half of the realizations at $y_{n}=4$ and the other half at $y_{n}=7$, either just before or just after breakthrough time. As for the heterogeneous model, the optimal policy was for surfactant injection for most state variable realizations at $y_{n}=4$. The histograms of the option value show that each reservoir model produces a unique distribution of the results, with the homogeneous model showing a higher number of realizations achieving a positive option value, whereas most realizations for the heterogeneous model achieve a negative option value.

Fig. 11 shows that more than $60 \%$ of realizations for the homogeneous model favored flexibility in surfactant injection compared with the static scenario. However, only $14 \%$ of realizations favored flexibility for the heterogeneous model. This significant 
Favoring Flexibility?

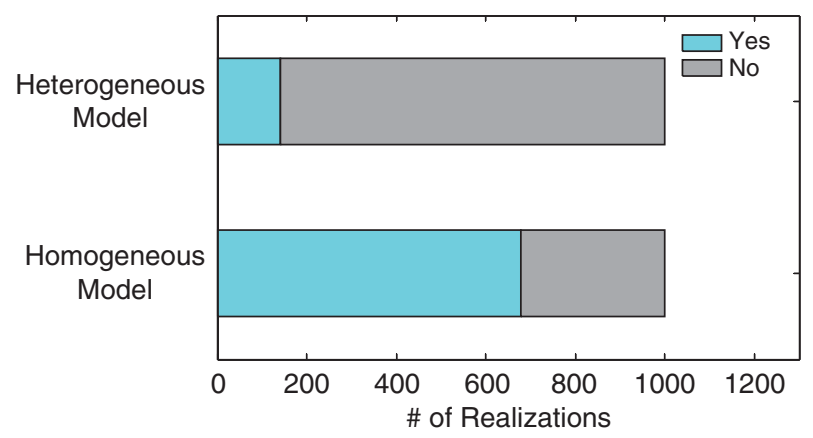

Fig. 11-Bar chart showing the number of realizations for both the homogeneous and heterogeneous reservoir models that favor flexibility.

discrepancy in optimal policy and value of flexibility illustrates the uniqueness of the response produced by the decision-making process for different reservoir models. Because the optimal policy for the heterogeneous model for most realizations was surfactant injection at the earliest decision node, this can be explained by the fact that surfactant injection performs better at earlier coarse realizations of the heterogeneity field rather than at the finer realizations (which occur at later decision nodes). This clearly depicts how our decisions are influenced by our assumptions on the realization of the permeability field with time.

The main reason for flexibility not being as beneficial in the heterogeneous model is the effect of channeling on the propagation of the surfactant bank through the reservoir. Consequently, we tend to get more channeling from the injector to the producer, which translates into more unswept oil-in other words, poorer sweep efficiency. This allows the surfactant to travel through these highly permeable channels and to not contact all the remaining oil. A possible remedy would be to add a mobility buffer to the surfactant-flooding process.

The effect of channeling on the performance of the surfactant flood is dependent on the well placement and the relationship of the wells to the structure of the heterogeneity. This can be illustrated with different quarter-five-spot well placements. Fig. 12 shows the results obtained from running the algorithm with the different possible placements with respect to the quarter-five-spot pattern. The original placement was found to achieve the best results (highest option value) compared with the other placements. Moreover, the effect of the general relationship between well placement and the heterogeneity structure on the results is illustrated. Because this relationship affects the channeling in the reservoir, other heterogeneous-reservoir models could produce different results compared with those produced by the example model used in this study. Furthermore, the use of other wellplacement patterns, such as line drive, or use of horizontal wells could also produce different results. Because the control variables (e.g., surfactant concentration, surfactant slug size) were fixed for both reservoir models, it is possible that if these variables were allowed to vary we would obtain different results for the heterogeneous model. However, adding different control strategies would increase the computational requirements of applying the algorithm because more reservoir simulations would be required. We emphasize that the main objective in this study was to demonstrate the applicability of the LSM method to a surfactant-EOR process with a simple decision.

The LSM method is well suited for combining technical and economic uncertainties. Its use depends on the design of the option or decision that is desired to be optimized. Once this is complete, the application of this algorithm is straightforward. By taking into consideration the uncertainty in the time series, this method provides us with the means to capitalize on the upside opportunities that these uncertainties present or helps us mitigate worsening circumstances. The algorithm depends on two main elements: the stochastic simulation of the input state variables and the regression process applied to solve the Bellman dynamic-programming principle. In the examples presented in this paper, we have used standard uncertainty distributions for the technical state variable and a standard mean-reverting stochastic price model for the main economic state variable (the oil price). Different distributions and models can easily be used with this algorithm without any major adjustments. Basis-function design is another important component in this algorithm, and it could have a significant influence on the results. However, for our examples here, a simple basis-function design was deemed sufficient (results were similar to those obtained with different polynomial families mentioned previously). Longstaff and Schwartz (2001) and Powell (2011) provide further discussion on basis-function design.

The regression method used was that of the standard leastsquares regression. Other advanced regression methods are also applicable with this algorithm, such as weighted least squares or generalized moment methods (Longstaff and Schwartz 2001).

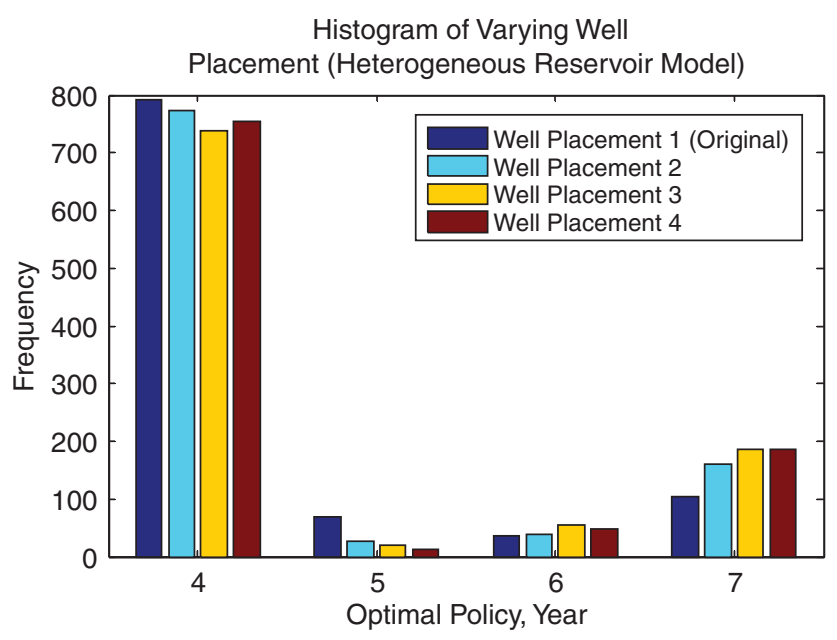

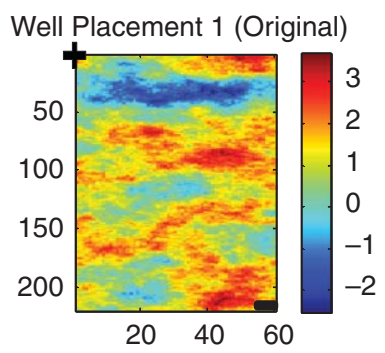

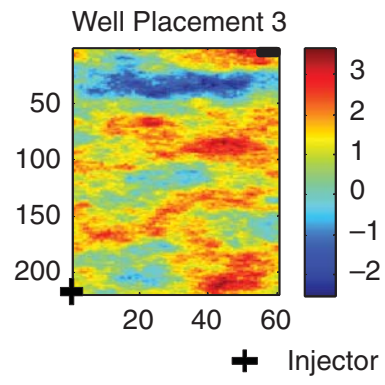

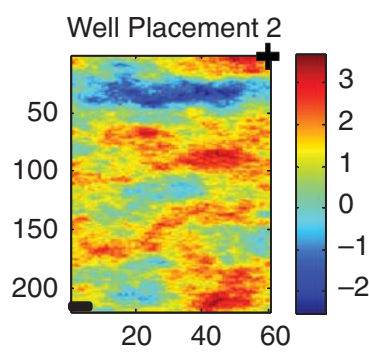

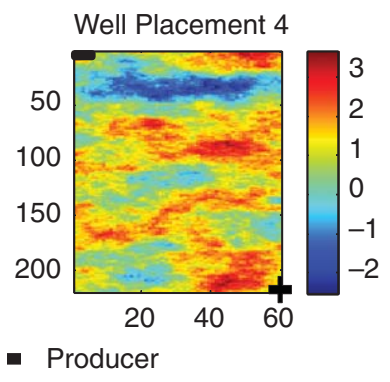

Fig. 12-Histogram of varying well placement on the heterogeneous reservoir model with $10^{3}$ realizations per run and plots of the different well placements considered. 

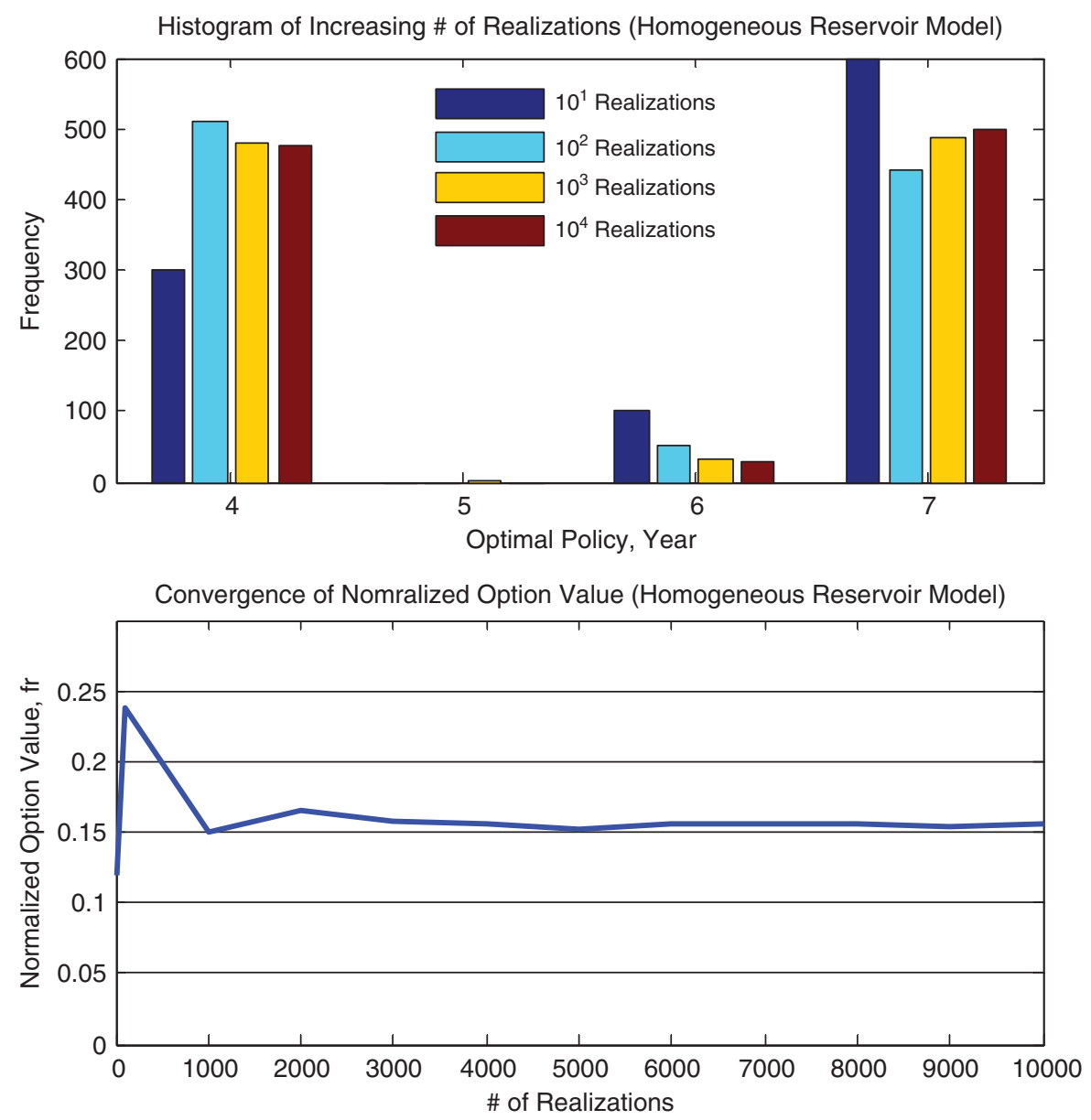

Fig. 13-Histogram of optimal policy (these have been adjusted to a basis of $10^{3}$ realizations to compare with the original run) and plot of convergence of the averaged normalized option value for the homogeneous reservoir model with increasing number of realizations. The averaged normalized option value is taken as the difference between the averaged (over all realizations) normalized option and the averaged normalized static scenarios; the normalization is with respect to the corresponding waterflood-scenario NPV (averaged over all realizations). A value greater than zero is considered to be favoring the options scenario, whereas a value less than zero favors the static scenario.

Sampling methods could also influence performance. Because the algorithm uses an approximation in estimating the continuation value, the policy obtained with this algorithm is considered to be a near-optimum rather than the global optimal policy. However, because the accuracy in estimating the continuation value increases, the results tend to converge to the global optimum (Jafarizadeh and Bratvold 2009). The initial sampling method for this algorithm is Monte Carlo sampling. This requires many realizations to achieve convergence, which necessitates great computational effort. For the simple examples in this study, it was possible to run the algorithm to the order of $10^{3}$ realizations; however, if we progress to $3 \mathrm{D}$ complex reservoir models, this could be problematic. Other sampling methods such as Latin hypercube sampling (McKay et al. 1979) could be easily used in the method.

Fig. 13 shows the convergence of the results as a function of the number of realizations used for each run and the optimal policy obtained. For the heterogeneous model, because of the large computational requirement needed to run $10^{4}$ realizations, multiple runs of $10^{3}$ realizations were performed and compared with the results of the initial heterogeneous model to test convergence (Fig. 14).

If we design more-sophisticated decision scenarios, this would require higher computational effort, as well. One possible way to enhance this algorithm for more-complex models is to design surrogate reservoir models, such as artificial neural networks (Karambeigi et al. 2011) or model approximations obtained from experimental designs (Cheong and Gupta 2005). Or we could enhance our sampling techniques to sample the high-probability regions of the distributions of the input technical state variables with methods such as polynomial chaos theory (Ghanem and Spanos 1991) or probabilistic collocation (Tatang et al. 1997). We hope that this would reduce computational requirements because reservoir simulation is the most demanding computational element in the LSM process.

It is also desired to incorporate more technical uncertainties, such as the effect of salinity on the surfactant performance, which will introduce a correlation with surfactant adsorption and consequently influence the recovery efficiency of the process. Ultimately, it is desired to incorporate dynamic control variables (such as varying the size and concentration of the injected chemical slug or varying the production constraints) in the evaluation method coupled with a highly discretized decision space (monthly or quarterly decisions rather than yearly decisions) to reflect more-realistic decision scenarios. The applicability of the LSM method is also possible for other EOR processes such as polymer, $\mathrm{CO}_{2}$, and thermal flooding. All that is needed is to define the relevant state variables, technical or economic, and to design the decision that is desired to be optimized, keeping in mind the constraint of the computational requirement.

\section{Conclusions}

This study introduced the LSM method as a simple yet powerful valuation technique for decision making under uncertainty for a specific EOR process. Different production strategies were evaluated given uncertainty in the reservoir and economic state variables. The method produced optimal policies conditional on the 

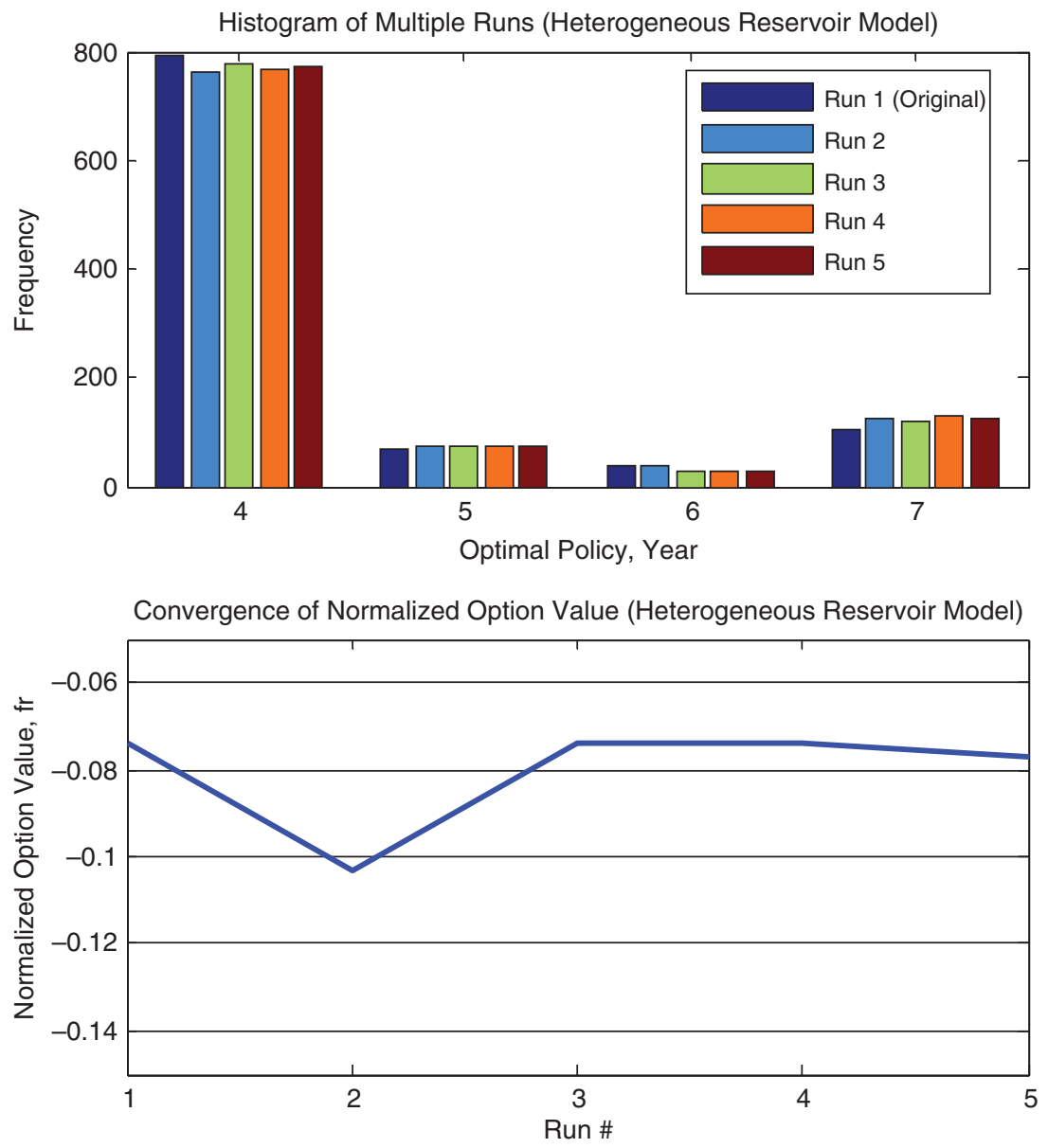

Fig. 14-Histogram of multiple runs on the heterogeneous reservoir model with $10^{3}$ realizations per run and plot of normalized option value per run. Optimal policies are similar to the original run (Run 1).

resolution of uncertainty at the decision time. This method was applied to a surfactant-flooding process of a quarter-five-spot pattern for two example reservoir models. The method was able to capture the impact of the relevant uncertainties and helped to determine better producing strategies given these uncertainties. In contrast to traditional valuation methods, the LSM method incorporates uncertainty in the time series in its evaluation and provides the ability to capture the value of upside opportunities. The accuracy of the results obtained by the LSM method was shown to be sufficient for the decision and reservoir models analyzed.

Although the results obtained are near-optimal policies, because they are rooted in regression, it does provide some insight into the effect of uncertainty on decision making. These insights could help in mitigating adverse circumstances should they arise or in capturing the upside potential if uncertainty resolves in a better than expected manner. The value of the option was determined about the no-option scenario assumed, which in this study was that of surfactant initiation at the start of field life. This introduced a bias into the value of flexibility. The form of bias varied on the basis of the permeability field used. On one hand, we have a pessimistic bias for the heterogeneous permeability field because injecting surfactant at the earliest possible time achieved the highest value, thus not favoring flexibility. Being afforded this opportunity, however, is rarely the case in real life. On the other hand, we have an optimistic bias for the homogeneous permeability field, where the highest value was achieved when surfactant was injected at a later time, thus favoring flexibility. This discrepancy in biases was attributed to the effect of channeling for the heterogeneous model used. We concluded that heterogeneity and different well-placement patterns affect the value of flexibility and optimal policy for different reservoir models. Furthermore, possible extensions that can be applied to the LSM method to value more-complex decisions for different EOR processes were discussed.

\section{Nomenclature}

$a=$ regression coefficient

$b=$ regression coefficient

$C=$ regression a constant

$D_{s}=$ surfactant adsorption, $1 \mathrm{bm} / \mathrm{lbm}$ rock

$E_{y^{*}}^{*}=$ expectation function

$\mathrm{F}=$ continuation function

$L_{j}=$ the $j$ th element in the orthonormal basis

$p=$ regression coefficient

$\operatorname{Pr}_{o}=$ oil price, USD $/ \mathrm{STB}$

$P r_{s}=$ surfactant cost, USD/STB surfactant

$P r_{w i}=$ cost of water injection, USD/STB

$P r_{w p}=$ cost of produced water, USD/STB

$q_{t}=$ range adjustment for Markov process

$r=$ discount factor

$S_{\text {orc }}=$ residual oil saturation to chemical flooding

$t=$ optimal time to begin the surfactant flood

$t(x)=$ optimal exercise time for a certain realization path

$W_{t}=$ a Brownian motion, $d W_{t} \sim N(0, \sqrt{d t})$

$(x)=$ one realization of the evolution of the state variables over a time horizon of 10 years

$x_{t}=$ state variable for Markov process

$X=$ total number of stochastic simulations

$y_{n}=$ decision time that is an element of the set of decision times, $Y=\left(y_{1}, \ldots, y_{n}\right)$

$Y=$ set of possible decision times

$\varepsilon_{t}=$ random variable for Markov process 
$\mu=$ the long-term mean to which the process reverts

$\rho=$ jump direction for Markov process

$\sigma=$ the risk premium

$\Sigma=$ the measure of the process volatility

$\xi=$ regression coefficient

$\lambda=$ measure of the speed of mean reversion

$\vartheta_{y_{n}}=$ information set $\left[\operatorname{Pr}_{o, y_{n}}(x)\right.$ and $\left.\operatorname{Pr}_{s, y_{n}}(x)\right]$ at time $y_{n}$

\section{References}

Amemiya, T. 1985. Advanced Econometrics. London: Basil Blackwell.

Anderson, G., Delshad, M., King, C., et al. 2006. Optimization of Chemical Flooding in a Mixed-Wet Dolomite Reservoir. Paper SPE 100082 presented at the SPE/DOE Symposium on Improved Oil Recovery, Tulsa, Oklahoma. 22-26 April. http://dx.doi.org/10.2118/100082-MS.

Babaei, M. and King, P. 2012. A Modified Nested-Gridding for Upscaling Downscaling in Reservoir Simulation. Transport Porous Med. 93 (3): 753-775. http://dx.doi.org/10.1007/s11242-012-9981-4.

Barua, J., Prescott, T. and Haldorsen, H. 1986. Financial and Technical Decision Making for Surfactant Flooding. Paper SPE 15074 presented at the SPE California Regional Meeting, Oakland, California, 2-4 April. http://dx.doi.org/10.2118/15074-MS.

Begg, S.H., Carter, R.R. and Dranfield, P. 1989. Assigning Effective Values to Simulator Gridblock Parameters for Heterogeneous Reservoirs. SPE Res Eng 4 (4): 455-463. http://dx.doi.org/10.2118/16754-PA.

Bensoussan. A. 1984. On the Theory of Option Pricing. Acta Appl. Math. 2 (2): 139-158. http://dx.doi.org/10.1007/BF00046576.

Bratvold, R. and Begg, S. 2010. Making Good Decisions. Richardson, Texas: Society of Petroleum Engineers.

Bratvold, R., Bickel, J. and Lohne, H. 2009. Value of Information in the Oil and Gas Industry: Past, Present, and Future. SPE Reservoir Evaluation \& Engineering 12 (4): 630-638. SPE 110378-PA. http://dx.doi.org/ 10.2118/110378-PA.

Brown, C. and Smith, P. 1984. The Evaluation of Uncertainty in Surfactant EOR Performance Prediction. Paper SPE 13237 presented at the SPE Annual Technical Conference and Exhibition, Houston, Texas, 16-19 September. http://dx.doi.org/10.2118/13237-MS.

Cheng, H., Shook, G., Taimur, M., et al. 2012. Interwell Tracer Tests to Optimize Operating Conditions for a Surfactant Field Trial: Design, Evaluation, and Implications. SPE Res Eval \& Eng 15 (2): 229-242. http://dx.doi.org/10.2118/144899-PA.

Cheong, Y. and Gupta, R. 2005. Experimental Design and Analysis Methods for Assessing Volumetric Uncertainties. SPE J. 10 (3): 324-335. http://dx.doi.org/10.2118/80537-PA.

Christie, M. and Blunt, M. 2001. Tenth SPE Comparative Solution Project: A Comparison of Upscaling Techniques. SPE Res Eval \& Eng 4 (4): 308-317. http://dx.doi.org/10.2118/72469-PA.

Costa, A., Schiozer, D., Moczydlower, P., et al. 2008. Use of Representative Models to Improve the Decision Making Process of Chemical Flooding in a Mature Field. Paper SPE 115442 presented at the SPE Russian Oil and Gas Technical Conference and Exhibition, Moscow, Russia, 28-30. http://dx.doi.org/10.2118/115442-MS.

Dang, C., Chen, Z., Nguyen, N., et al. 2011. Develpment of Isotherm Polymer/Surfactant Adsorption Models in Chemical Flooding. Paper SPE 147872 presented at the SPE Asia Pacific Oil and Gas Conference and Exhibition, Jakarta, Indonesia, 20-22 September. http://dx.doi.org/ 10.2118/147872-MS.

Dias, M. 2004. Monte Carlo Simulation of Stochastic Processes, http:// www.puc-rio.br/marco.ind/sim_stoc_proc.html (accessed 17 July 2012).

Dixit, A. and Pindyck, R. 1994. Investment Under Uncertainty. Princeton, New Jersey: Princeton University Press.

DOE. 1986. Scientific Software-Intercomp: Supporting Technology for Enhanced Oil Recovery - Chemical Predictive Model, VenezuelaMEM/USA-DOE Fossil Energy. Report III-5 (December 1986).

ECLIPSE Reservoir Engineering Software. 2010. Schlumberger, http:// www.slb.com/services/software/reseng/eclipse.aspx. (accessed 11 January 2010).

ECLIPSE Technical Description, London, UK, Version 2010.1. 2010. Schlumberger.

Energy Information Agency (EIA). 2012. NYMEX Futures Price Data. http://www.eia.gov/dnav/pet/pet_pri_fut_s1_d.htm (accessed 10 July 2012).
Gamba, Andrea. 2002. An Extension of Least Squares Monte Carlo Simulation for Multi-options Problems. Oral presentation given at the 6th Annual Real Options Conference, Paphos, Cyprus. 4-6 July.

Gerbacia, W. 1978. The Evaluation of Surfactant Systems for Oil Recovery Using Statistical Design Principles and Analysis. Paper SPE 7070 presented at the SPE Symposium on Improved Methods of Oil Recovery, Tulsa, Oklahoma, 16-17 April. http://dx.doi.org/10.2118/7070MS.

Ghanem, R. and Spanos, P. 1991. Stochastic Finite Elements: A Spectral Approach. New York: Springer-Verlag.

Gittler, W. and Krumrine, P. H. 1985. A Novel Approach for Risk Assessment in Chemical EOR Projects. Paper SPE 13767 presented at the SPE Hydrocarbon Economics and Evaluation Symposium, Dallas, Texas, 14-15 March. http://dx.doi.org/10.2118/13767 -MS.

Grayson, C. 1962. The Calculus of Common Sense. Paper SPE 309 presented at the Annual Meeting of the American Institute of Mining, Metallurgical, and Petroleum Engineers, Los Angeles, California, 8-10. http://dx.doi.org/10.2118/309-MS.

Hankins, N. P. and Harwell, J. H. 1996. Case Studies for the Feasibility of Sweep Improvement in Surfactant-Assisted Waterflooding. J. Pet. Sci. Eng. 17 (1): 41-62. http://dx.doi.org/10.1016/S0920-4105(96)000551 .

Hirasaki, G., Miller, C. and Puerto, M. 2011. Recent Advances in Surfactant EOR. SPE J. 16 (4): 889-907. http://dx.doi.org/10.2118/115386PA.

Jafarizadeh, B. and Bratvold, R. B. 2009. Taking Real Options Into the Real World: Asset Valuation Through Option Simulation. Paper SPE 124488 presented at the SPE Annual Technical Conference and Exhibition, New Orleans, Louisiana, 4-7 October. http://dx.doi.org/ $10.2118 / 124488$.

Karambeigi, M., Zabihi, R. and Hekmat, Z. 2011. Neuro-Simulation Modeling of Chemical Flooding. J. Pet. Sci. Eng. 78 (2): 208-219. http:// dx.doi.org/10.1016/j.petrol.2011.07.012.

Karatzas, I. 1988. On the Pricing of American Options. Appl. Math. Opt. 17 (1): 37-60. http://dx.doi.org/10.1007/BF01448358.

Kossack, C. and Bilhartz, H. 1976. The Sensitivity of Micellar Flooding to Reservoir Heterogeneities. Paper SPE 5808 presented at the SPE Improved Oil Recovery Symposium, Tulsa, Oklahoma, 22-24 March. http://dx.doi.org/10.2118/5808-MS.

Lake, L. W. 1989. Enhanced Oil Recovery. Englewood Cliffs, New Jersey: Prentice Hall.

Lohne, A. and Fjelde, I. 2012. Surfactant Flooding in Heterogeneous Formations. Paper SPE 154178 presented at the SPE Improved Oil Recovery Symposium, Tulsa, Oklahoma, 14-18 April. http://dx.doi.org/ 10.2118/154178-MS.

Longstaff, F. A. and Schwartz, E. S. 2001. Valuing American Options by Simulation: A Simple Least-Squares Approach. Rev. Financ. Stud. 14 (1): 113-147. http://dx.doi.org/10.1093/rfs/14.1.113.

Maerker, J. and Gale, W. 1992. Surfactant Flood Process Design for Loudon. SPE Res Eval \& Eng 7 (1): 36-44. http://dx.doi.org/10.2118/ 20218-PA.

Manrique, E., Izadi, M., Kitchen, C., et al. 2009. Effective EOR Decision Strategies With Limited Data: Field Cases Demonstration. SPE Res Eval \& Eng 12 (4): 551-561. http://dx.doi.org/10.2118/113269PA.

Matlab $^{\circledR}$, version 7.14. 2012. Natick, Massachusetts: The Mathworks, Inc.

McKay, M. D., Beckman, R. J. and Conover, W. J. 1979. A Comparison of Three Methods for Selecting Values of Input Variables in the Analysis of Output from a Computer Code. Technometrics 21 (2): 239-245. http://dx.doi.org/10.2307/1268522.

Moreno, M. and Navas, J. 2003. On the Robustness of Least-Squares Monte Carlo (LSM) for Pricing American Derivatives. Review of Derivatives Research 6 (2): 107-128. http://dx.doi.org/ 10.1023/ A:1027340210935.

Pope, G., Wang, B. and Tsaur, K. 1979. A Sensitivity Study of Micellar/ Polymer Flooding. SPE J. 19 (6): 357-368. http://dx.doi.org/10.2118/ 7079-PA.

Puerto, M., Hirasaki, G., Miller, C., et al. 2012. Surfactant Systems for EOR in High-Temperature, High-Salinity Environments. SPE J. 17 (1): 11-19. http://dx.doi.org/10.2118/129675-PA. 
Powell, W. B. 2011. Approximate Dynamic Programming: Solving the Curses of Dimensionality. Princeton, New Jersey: John Wiley and Sons.

Raterman, K., Kremesec, V. and Suffridge, F. 1988. Evaluation of LowConcentration Surfactant Flooding in the Absence of Mobility Control Agents. Paper SPE 17394 presented at the SPE Enhanced Oil Recovery Symposium, Tulsa, Oklahoma, 16-21 April. http://dx.doi.org/ 10.2118/17394-MS.

Reppert, T. R., Bragg, J. R., Wilkinson, J. R., et al. 1990. Second Ripley Surfactant Flood Pilot Test. Paper SPE 20219 presented at the SPE/ DOE Enhanced Oil Recovery Symposium, Tulsa, Oklahoma, 22-25 April. http://dx.doi.org/10.2118/20219-MS.

Sanz, C. and Miller, M. 1994. A Decision Analysis Approach to the Design of a Chemical Flooding Process. Paper SPE 27036 presented at the SPE Latin American/Caribbean Petroleum Engineering Conference, Buenos Aires, Argentina, 27-29 April. http://dx.doi.org/10.2118/ 27036-MS.

Smith, W. 2010. On the Simulation and Estimation of the Mean-Reverting Ornstein-Uhlenbeck Process. http://commoditymodels.files.wordpress. com/2010/02/estimating-the-parameters-of-a-mean-reverting-ornsteinuhlenbeck-process1.pdf (downloaded 9 January 2012).

Solairaj, S., Britton, C., Kim, D., et al. 2012. Measurement and Analysis of Surfactant Retention. Paper SPE 154247 presented at the SPE Improved Oil Recovery Symposium, Tulsa, Oklahoma, 14-18 April. http://dx.doi.org/10.2118/154247-MS.

STARS Reservoir Simulator. 2012. Computer Modelling Group LTD. http://www.cmgl.ca/software/soft-stars.

Tatang, M., Pan, W., Prinn, R., et al. 1997. An Efficient Method for Parametric Uncertainty Analysis of Numerical Geophysical Models. $J$. Geophys. Res. 102 (18): 21925-21932. http://dx.doi.org/ 10.1029/ 97JD01654.

Thomas, S. 2006. Chemical EOR: The Past - Does It Have a Future? Paper SPE 108828 based on a speech presented as a Distinguished Lecture during the 2005-2006 season.

Tomich, J., Laplante, L. and Snow, T. 1987. Technical and Economic Complexities Associated with Surfactant Flooding. Paper WPC 22236 presented at $12^{\text {th }}$ World Petroleum Congress, Houston, Texas, 26 April-1 May.

Trantham, J. 1983. Prospects of Commercialization, Surfactant/Polymer Flooding, North Burbank Unit, Osage County, OK. J Pet Technol 35 (5): 872-880. http://dx.doi.org/10.2118/9816-PA.

Uhlenbeck, G. and Ornstein, L. 1930. On the Theory of the Brownian Motion. Phys. Rev. 36 (5): 823-841. http://link.aps.org/doi/10.1103/ PhysRev.36.823.

Warren, J. and Price, H. 1961. Flow in Heterogeneous Porous Media. SPE J. 1 (3): 153-169. http://dx.doi.org/10.2118/1579-G.

White, H. 1984. Asymptotic Theory for Econometricians. New York: Academic Press.

Willigers, B. J. A. and Bratvold, R. B. 2009. Valuing Oil and Gas Options by Least-Squares Monte Carlo Simulation. SPE Proj Fac \& Const 4 (4): 146-155. http://dx.doi.org/10.2118/116026-PA.

Wyatt, K., Pitts, M. and Surkalo, H. 2008. Economics of Field Proven Chemical Flooding Technologies. Paper SPE 113126 presented at the SPE/DOE Symposium on Improved Oil Recovery, Tulsa, Oklahoma, 20-23 April. http://dx.doi.org/10.2118/113126-MS.

\section{Appendix A-Reservoir and Surfactant Properties}

See Tables A-1 through A-6.

\section{TABLE A-1-HOMOGENEOUS-RESERVOIR SIMULATION-MODEL PROPERTIES}

\begin{tabular}{|lc|} 
Grid & $10 \times 10 \times 10$ Cells \\
\hline Dimensions & $466 \times 466 \times 25 \mathrm{ft}(142 \times 142 \times 8.2 \mathrm{~m})$ \\
$k_{h}$ & $100 \mathrm{md}$ \\
$k_{v}$ & $10 \mathrm{md}$ \\
Porosity, \% & 20 \\
Top of reservoir & $4,920 \mathrm{ft}(1500 \mathrm{~m})$ \\
\hline
\end{tabular}

TABLE A-2-IMMISCIBLE RELATIVE PERMEABILITY DATA

\begin{tabular}{lccc|}
$S_{w}$ & $k_{r w}$ & $k_{\text {row }}$ & $p_{\text {cwo }}$ \\
\hline 0.2 & 0 & 0.9 & 50 \\
0.22 & 0 & 0.803 & 45 \\
0.3 & 0.001 & 0.487 & 25 \\
0.4 & 0.009 & 0.221 & 12.5 \\
0.5 & 0.045 & 0.078 & 6.3 \\
0.6 & 0.154 & 0.014 & 2.5 \\
0.7 & 0.387 & 0.001 & 1.3 \\
0.73 & 0.48 & 0 & 1.1 \\
0.8 & 0.8 & 0 & 0.8 \\
\hline
\end{tabular}

Data source is ECLIPSE Technical Description 2010.1.

TABLE A-3-SURFACTANT VISCOSITY

\begin{tabular}{|lc|}
$\begin{array}{l}\text { Surfactant } \\
\text { Concentration }(\mathrm{Ibm} / \mathrm{STB})\end{array}$ & $\begin{array}{c}\text { Surfactant Water } \\
\text { Viscosity }(\mathrm{cp})\end{array}$ \\
\hline 0 & 1.0 \\
0.1 & 1.1 \\
0.2 & 1.2 \\
0.8 & 1.3 \\
\hline Data source is ECLIPSE Technical Description 2010.1. \\
\hline
\end{tabular}

TABLE A-4-SURFACTANT/OIL SURFACE TENSION

\begin{tabular}{|lc|}
$\begin{array}{l}\text { Surfactant } \\
\text { Concentration } \\
\text { (lbm/STB) }\end{array}$ & $\begin{array}{c}\text { Surfactant Water/ } \\
\text { Oil Surface } \\
\text { Tension (lbf/in.) }\end{array}$ \\
\hline 0 & 100 \\
0.1 & 50 \\
0.2 & 10 \\
0.5 & 0 \\
\hline Data source is ECLIPSE Technical Description 2010.1. \\
\hline
\end{tabular}

\begin{tabular}{|c|c|}
\hline \multicolumn{2}{|c|}{ TABLE A-5-CAPILLARY DESATURATION CURVE } \\
\hline $\log _{10}$ (Capillary Number) & Miscibility Function \\
\hline-9 & 0 \\
\hline 2 & 0 \\
\hline 5 & 1 \\
\hline 10 & 1 \\
\hline
\end{tabular}

TABLE A-6-RESERVOIR-SIMULATION CONSTRAINTS

Constraint Limit Value

Bottomhole-pressure minimum, psia 250

Oil rate, $B / D$

Water cut, \% 20 (maximum)

$W_{\text {inj }}$ maximum pressure, psia 
Ali Alkhatib is currently a fourth-year PhD candidate at Imperial College London. He previously worked as a reservoir engineer at Saudi Aramco. Alkhatib's interests include approximate dynamic programming, decision making under uncertainty, and quantification of uncertainty for chemical-EOR processes. He holds a BSc degree with honors from the University of Edinburgh in chemical engineering and an MSc degree with merit in petroleum engineering from Imperial College London.

Masoud Babaei is a research associate in the Department of Earth Science and Engineering at Imperial College London. $\mathrm{He}$ is currently working on $\mathrm{CO}_{2}$ storage and sequestration. Babaei holds a PhD degree from Imperial College London. His $\mathrm{PhD}$ research was on upscaling of reservoir models. Babaei also holds an MS degree from Sharif University, Iran, and a BS degree from Petroleum University of Technology, Iran, both in reservoir engineering.

Peter King is currently a professor of petroleum engineering at Imperial College London. He previously spent 17 years with BP at the company's technology center in Sunbury-on-Thames, where he worked on a wide variety of subjects applying methods of mathematical physics to reservoir characterization and modeling. King's recent work focuses on applying stochastic search algorithms to optimizing business decisions, with particular interest in decision making in the presence of uncertainty. $\mathrm{He}$ holds a PhD degree in theoretical statistical physics from Cambridge University.

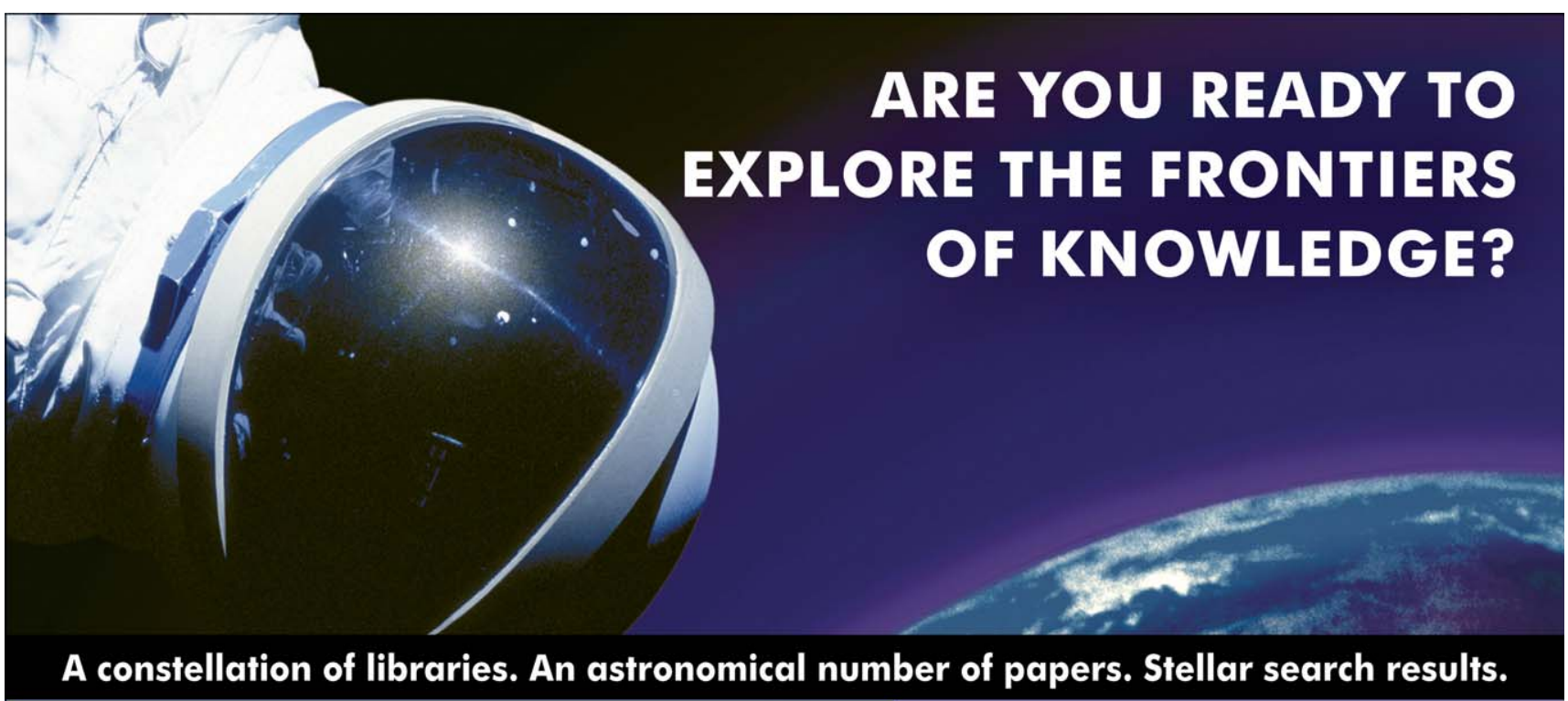

OnePetro brings together specialized technical libraries serving the oil and gas industry into one, easy-to-use website-allowing you to search and download documents from multiple professional societies in a single transaction. With more than 150,000 technical papers, one search can help you locate the solutions you need. A range of

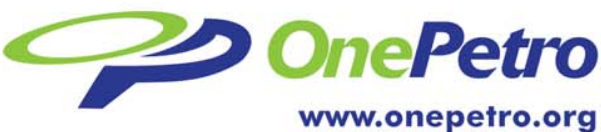
subscription options make accessing the results easy.

www.onepetro.org

Have you explored what OnePetro has to offer? 